\title{
MILLIMETER-WAVE CHANNEL MEASUREMENT AT 73GHZ AND 81GHZ
}

\author{
by
}

Changyu Guo

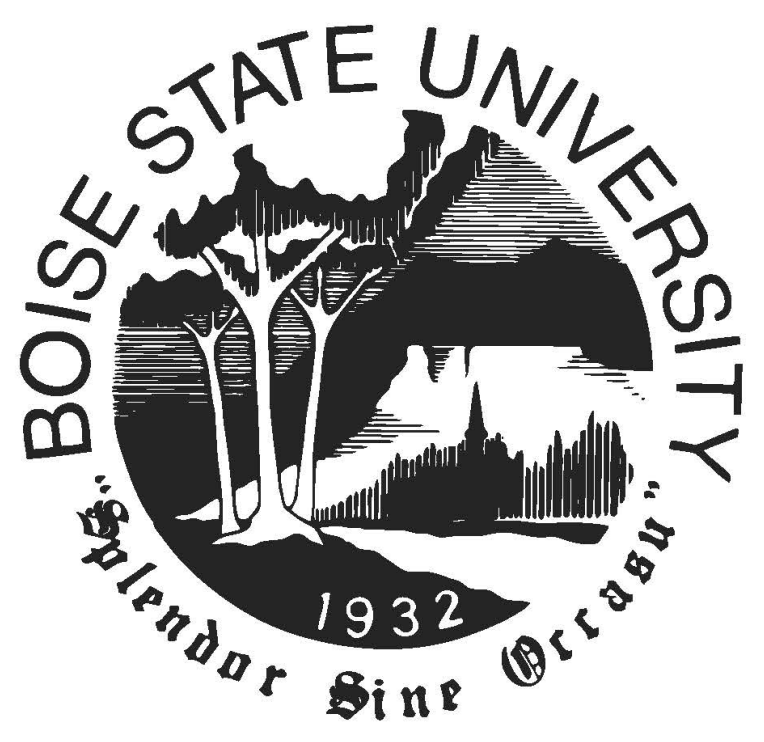

\author{
A thesis \\ submitted in partial fulfillment \\ of the requirements for the degree of \\ Master of Science in Electrical and Computer Engineering \\ Boise State University
}

May 2021 
(C) 2021

Changyu Guo

ALL RIGHTS RESERVED 


\section{BOISE STATE UNIVERSITY GRADUATE COLLEGE DEFENSE COMMITTEE AND FINAL READING Approval}

of the thesis submitted by

Changyu Guo

Thesis Title: Millimeter-Wave Channel Measurement at $73 \mathrm{GHz}$ and $81 \mathrm{GHz}$

Date of Oral Examination: $\quad 23$ March 2021

The following individuals read and discussed the thesis submitted by student Changyu Guo, and they evaluated the student's presentation and response to questions during the final oral examination. They found that the student passed the final oral examination.

Hani Mehrpouyan, Ph.D. Chair, Supervisory Committee

Nader Rafla, Ph.D. Member, Supervisory Committee

Hao Chen, Ph.D. Member, Supervisory Committee

The final reading approval of the thesis was granted by Hani Mehrpouyan, Ph.D., Chair of the Supervisory Committee. The thesis was approved by the Graduate College. 
To my supportive family and friends. 


\section{ACKNOWLEDGMENTS}

It has been a great journey at Boise State University while perusing my Master's degree.

Foremost, I would like to thank my adviser Dr. Mehrpouyan, who has supported me from the end of my undergrad study to the end of my Master's study. He has always been supportive and pointing me in directions when I was lost. This thesis would not have been possible without his guidance.

I would like to thank my committee members, Dr. Chen and Dr. Rafla who served also as my mentors when I needed them.

I would like to thank my colleagues, especially Mahfuza Khatun, for the help and guidance.

I would like to thank Nozhan Hosseini, Dr. Matolak from the University of South Carolina, Kairui Du, Ozgur Ozdemir, and Dr.Guvenc from North Carolina State University. We worked together on building materials attenuation measurement, which is presented in section 3.2.

Last but not least, I would like to thank my parents and my friends for the company and help along the way. 


\begin{abstract}
The abundant spectrum available at mmWave band can provide high capacity, high throughput, and low latency. In this thesis, we present experimental measurements for 73 and $81 \mathrm{GHz}$ mmWave bands. Wideband propagation measurements were performed at the Boise Airport concourse $\mathrm{C}$ area and tarmac for both line-of-sight (LOS) and non-line-of sight (NLOS) scenarios. Power delay profiles were recorded and analyzed with close-in free space reference path loss models and floating intercept path loss models. In addition, building material attenuation at 28, 73, and $91 \mathrm{GHz}$ is presented. Measurements at $73 \mathrm{GHz}$ for wideband and narrowband signal are performed with directional antennas. Moreover, wideband spatial fading measurements were performed at the Boise State University Micron Engineering Building and Boise Airport. The power delay profiles are recorded and analyzed with Rayleigh, Ricean, and log-normal models. Large scale path loss parameters at the airport, material attenuation and small scale fading parameters were computed. The results can help researchers and network designers in simulation and design of mmWave wireless networks.
\end{abstract}




\section{TABLE OF CONTENTS}

ABSTRACT $\ldots \ldots \ldots \ldots \ldots \ldots \ldots \ldots \ldots \ldots \ldots \ldots \ldots \ldots \ldots \ldots \ldots$

LIST OF TABLES $\ldots \ldots \ldots \ldots \ldots \ldots \ldots \ldots \ldots \ldots$ ix

LIST OF FIGURES $\ldots \ldots \ldots \ldots \ldots \ldots \ldots \ldots \ldots \ldots \ldots \ldots \ldots \ldots \ldots \ldots$

LIST OF ABBREVIATIONS $\ldots \ldots \ldots \ldots \ldots \ldots \ldots \ldots \ldots \ldots \ldots \ldots \ldots \ldots$

1 Introduction $\ldots \ldots \ldots \ldots \ldots \ldots \ldots \ldots \ldots \ldots \ldots \ldots \ldots \ldots \ldots \ldots \ldots$

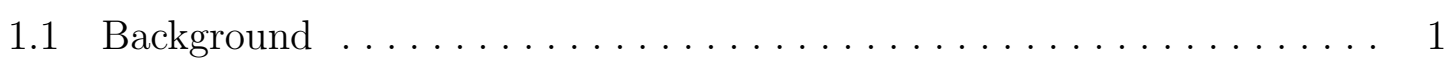

1.2 Importance of Channel Measurements . . . . . . . . . . . . . 3

1.3 Literature Review $\ldots \ldots \ldots \ldots \ldots \ldots \ldots \ldots \ldots \ldots \ldots \ldots$

1.4 Contribution and structure of the thesis ............... 5

2 Overview of Channel Modeling $\ldots \ldots \ldots \ldots \ldots \ldots \ldots \ldots \ldots$

2.1 Radio Wave Propagation $\ldots \ldots \ldots \ldots \ldots \ldots \ldots \ldots \ldots$

2.2 Propagation Modeling $\ldots \ldots \ldots \ldots \ldots \ldots \ldots \ldots \ldots \ldots \ldots \ldots \ldots \ldots$

2.2.1 Large scale propagation channel effects............. 8

2.2.2 Small scale propagation channel effects.............. 12

3 Measurement campaign and analysis $\ldots \ldots \ldots \ldots \ldots \ldots \ldots \ldots$

3.1 Large Scale fading Measurement at Boise Airport ............. 13

$3.1 .1 \quad$ System $\operatorname{setup} \ldots \ldots \ldots \ldots \ldots \ldots \ldots \ldots \ldots \ldots \ldots \ldots$ 
3.1 .2 Measurement Environment . . . . . . . . . . . . . . . . 14

3.1 .3 Measurement Procedure . . . . . . . . . . . . . . . . 16

3.1.4 Data Analysis and Result . . . . . . . . . . . . . . . . 17

3.2 Attenuation of Different Building Materials . . . . . . . . . . . . . 21

3.2 .1 System $\operatorname{setup} \ldots \ldots \ldots \ldots \ldots \ldots \ldots \ldots \ldots \ldots \ldots$

3.2.2 Measurement Procedure . . . . . . . . . . . . . . . . . . 23

3.2 .3 Data Analysis Result . . . . . . . . . . . . . . 26

3.3 Small scale measurement at Boise State University and Boise Airport. . 27

3.3 .1 System Setup . . . . . . . . . . . . . . . . . 27

3.3.2 Measurement Environment and Procedure ............. 28

3.3.3 Data Analysis Result . . . . . . . . . . . . . . . . . . 29

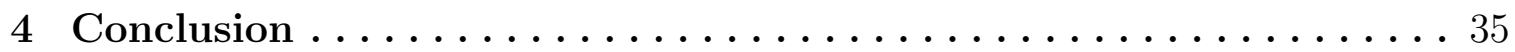

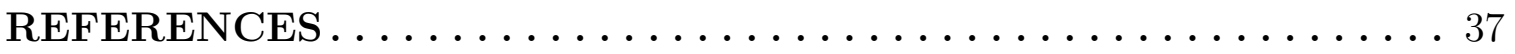

A Linear Rail System ....................... 41

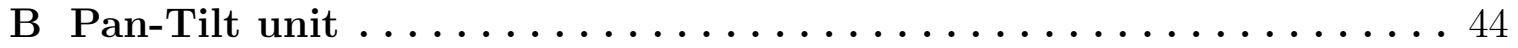

C Additional figures for Materials Attenuation Measurement . . . . . 47 


\section{LIST OF TABLES}

3.1 Hardware Specifications of 73 and $81 \mathrm{GHz}$ Channel Measurements [1] . 13

3.2 Directional close-in free space reference distance $\left(d_{0}=1 \mathrm{~m}\right)$ path loss models EQ.(2.6) for LOS and NLOS. $n$ is the PLE and $\sigma$ is the standard deviation of the shadow factor(a zero mean random Gaussian variable) [1]19

3.3 Directional floating intercept path loss models for LOS and NLOS. $\alpha$ is the floating intercept, $\beta$ is the slope of the MMSE fitted line and $\sigma$ is the standard deviation of the shadow factor $[1] \ldots \ldots \ldots \ldots$

3.4 Hardware Specifications for $73 \mathrm{GHz}$ Measurement . . . . . . . . . . . . 21

3.5 Average specific attenuation with standard deviation at $28 \mathrm{GHz}$ (NCSU), $73 \mathrm{GHz}(\mathrm{BSU})$ and $91 \mathrm{GHz}$ (USC) for wide band measurements. Cinder block attenuation at 91 and $73 \mathrm{GHz}$ is only narrow band result due to

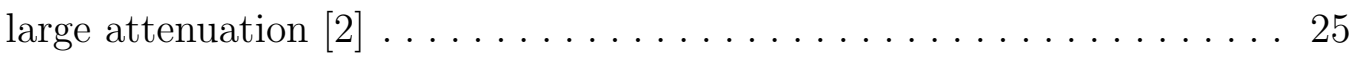

3.6 Hardware Specifications for Small Scale Measurement . . . . . . . . . . . 27 


\section{LIST OF FIGURES}

3.1 System diagram of transmitter $[1] \ldots \ldots \ldots \ldots \ldots \ldots \ldots$

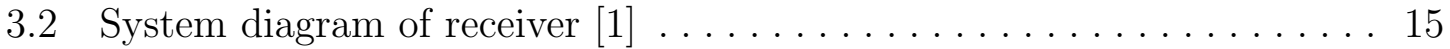

3.3 Layout of concourse C area at Boise Airport, with LOS and NLOS TX

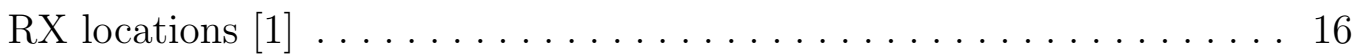

3.4 Map of tarmac at Boise Airport, showing TX and RX locations [1] . . . 16

3.5 $73 \mathrm{GHz}$ and $81 \mathrm{GHz}$ indoor directional CI $\left(d_{0}=1\right)$ and FI path loss models with TX antenna heights of $1.5 \mathrm{~m}$ and RX antenna heights of $1.2 \mathrm{~m}$ at Boise Airport concourse C. Blue dots are LOS path loss values, green squares are obstructed NLOS path loss values. [1] . . . . . . . . 18

3.6 $73 \mathrm{GHz}$ and $81 \mathrm{GHz}$ outdoor directional CI $\left(d_{0}=1\right)$ and FI path loss models with TX antenna heights of $1.3 \mathrm{~m}$ and RX antenna heights of $1.2 \mathrm{~m}$ at Boise Airport tarmac. Blue dots are LOS path loss values, green squares are obstructed NLOS path loss values. [1] . . . . . . . . . 18

3.7 Geometry for determining projection area and material dimensions

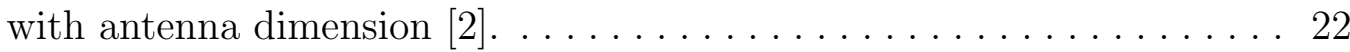

3.8 Measurement picture for glass, with RX on the left and TX on the right. In the measurement a smaller dimension antenna was used for

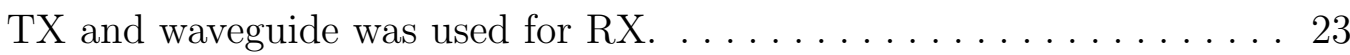

3.9 Measurement diagram, top view, with material in the middle, RX locations on the left, and TX locations on the right. 
3.10 Average attenuation versus frequency at $28,73,91 \mathrm{GHz}$ for all test

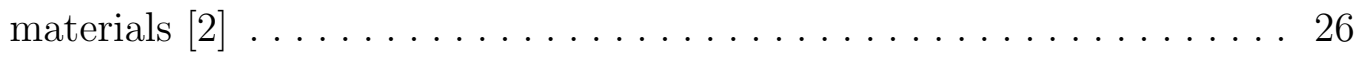

3.11 image of $73 \mathrm{GHz}$ small scale indoor measurement . . . . . . . . . . . 29

3.12 a: Layout of the small scale measurement office environment, showing office and cubicles. b: Layout of the small scale measurement outdoor environment, not showing the airplane connected to the jetbridge during the measurement, and a truck blocking the NLOS TX-RX link. . 30

3.13 a: Power delay profiles with various RX antenna pointing angle, outdoor LOS at Boise Airport, $\mathrm{fc}=73 \mathrm{GHz} d=7.4 \mathrm{~m}$ b: Angular power profile of $73 \mathrm{GHz}$ signal, outdoor LOS at Boise Airport, $\mathrm{d}=7.4 \mathrm{~m} \ldots . .31$

3.14 CDFs of the, a: $73 \mathrm{Ghz}$ and b: $81 \mathrm{GHz}$, small scale fading of individual path amplitude relative to rms amplitude, in LOS indoor environment. 32

3.15 CDFs of the, a: $73 \mathrm{Ghz}$ and b: $81 \mathrm{GHz}$, small scale fading of individual path amplitude relative to rms amplitude, in LOS outdoor airport

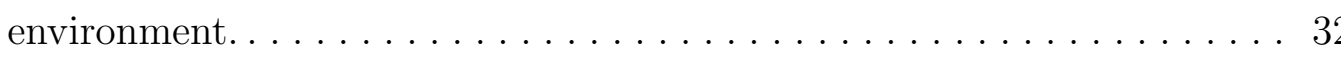

3.16 CDFs of the, a: $73 \mathrm{Ghz}$ and b: $81 \mathrm{GHz}$, small scale fading of individual path amplitude relative to rms amplitude, in NLOS outdoor airport

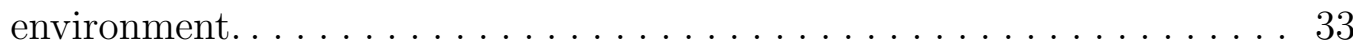

A.1 Linear Rail Module. . . . . . . . . . . . . . . . . . . . . . . . . . . 42

A.2 Linear rail with Gimbal and RX mounted on cart . . . . . . . . . . . 43

B.1 PTU Control System Diagram . . . . . . . . . . . . . . . . . 45

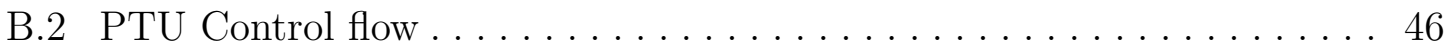


C.1 Specific Attenuation versus Frequency for different materials. top left: plywood, top right: glass, middle left: drywall, middle right: concrete, bottom: ceiling tile $[2] \ldots \ldots \ldots \ldots \ldots \ldots \ldots \ldots \ldots \ldots \ldots$ 


\section{LIST OF ABBREVIATIONS}

FDMA - Frequency-division multiple access

TDMA - Time-division multiple access

CDMA - Code-division multiple access

FDD - Frequency division duplex

GSM - Global System for Mobile

WLAN - Wireless local area network

LOS - Line of Sight

NLOS - None line of Sight

PLE - Path Loss Exponent

AWG - Arbitrary Waveform Generator

FSPL - Free Space Path Loss

TX - Transmitter

RX - Receiver

mmWave - Millimeter wave

PDP - Power Delay Profile

CI - Close-in free space reference distance

FI - Floating Intercept 
BSU - Boise State University

USC - University of South Carolina

NCSU - North Carolina State University

PTU - Pan-Tilt Unit

CDF - Cumulative distribution functions 


\section{CHAPTER 1}

\section{INTRODUCTION}

\subsection{Background}

Millimeter wave (mmWave) wireless communication systems represent the evolution of modern communications. The radio telegraph system was invented by Marconi in the early 1900s. Since then, the wireless industry has expanded from point-to-point communication, to broadcast system, and finally to wireless networks.

First generation $(1 \mathrm{G})$ wireless communication service was introduced in the 1980 s. $1 \mathrm{G}$ used analog modulation and frequency division multiple access (FDMA) to support multiple users. Second generation cellular networks (2G) were deployed in the 1990s, with digital modulation and TDMA/FDD and CDMA/FDD as multiple access techniques. There were still different systems, code division multiple access (CDMA) standards, and TDMA standards, i.e. Global System for Mobile (GSM), Personal Digital Cellular (PDC), and interim standard 136 (IS-136). Third generation cellular networks $(3 \mathrm{G})$ rolled off in 2000s. 3G systems promise wireless access in the way that was never possible before. It provides multi-megabit internet access, voice over IP (VoIP) protocol, unparalleled network capacity and more. The International Telecommunication Union (ITU) worked on the development of a global broadband multimedia telecommunication, called International Mobile Telephone 2020 (IMT-2000). From the 2G era, users were split into GSM/PDC/IS-136 
and CDMA. GSM/PDC/IS-136 leads to Wideband CDMA (W-CDMA) also known as Universal Mobile Telecommunication Service (UMTS), and CDMA evolves to cdma2000. Stepping in to $4 \mathrm{G}$, mobile broadband service became the biggest focus, with tough requirements on data rates, latency and capacity. It also merges all users into one universal accepted standard. Today, many service providers started $5 \mathrm{G}$ network service, and more and more mobile devices are being purchased on the market.

The connectivity provided by wireless networks has become an inseparable part to modern society. Other than traditional mobile services, emerging applications, such as the Internet of things (IOT) and vehicular and aerospace applications, of wireless communication are also rapidly growing. The increased growth brings overwhelming demand on higher capacity. At mmWave, available spectrum is unparalleled compared to sub-6 GHz cellular networks and wireless local area networks (WLAN). Over $20 \mathrm{GHz}$ of spectrum is available in 28, 38, and $72 \mathrm{GHz}$ alone [3].

Other than the significant jump on the spectrum, which offers higher data rates and capacities, there are several other advantages of using mmWave signals. Due to its small wavelength, antenna size becomes small, high gain and high directional steerable antenna can be used, which leads to potentially new spatial processing techniques, such as massive MIMO and spatial modulation $[4,5]$. mmWave signals also have higher path loss compared with current $700 \mathrm{MHz}$ to $2.6 \mathrm{GHz}$ frequencies. As cell coverage area gets smaller, the greater path loss becomes useful for cooperative architectures such as spatial reuse, interference mitigation, relays, and heterogeneous networks.

In the aviation field, air traffic is growing from increasing air transportation and unmanned aircraft systems. NASA is funding research efforts to develop air to ground 
and airport surface communication systems with higher capacity and service quality.

\subsection{Importance of Channel Measurements}

Radio channels are a fundamental element in communication systems. Modern wireless communication system standardization begins with characterizations of the wireless channel in different environments and over the system it will operate. A channel can consist of a number of components that cause the transmit signal to go through reflection, refraction, and diffraction. Thus a signal transmit through a channel could face challenges such as path loss, delay, phase shift, shadowing and interference. With technical understanding of the channel, engineers and researchers may study new methods for air interface, new signal processing techniques, and new architectural designs.

\subsection{Literature Review}

Intensive simulations and measurements have been presented to better understand mmWave propagation characteristics [6-10].

T.S. Rappaport et al. from New York University presents comprehensive research in mmWave channel measurement and modeling. In [6], they conducted measurements for reflection and penetration loss in and around buildings in New York City at $28 \mathrm{GHz}$. They utilized a sliding correlator channel sounder and measured received power from various building materials. Materials they tested in the paper include tinted glass, clear glass, brick, and wall. They measured outdoor materials such as tinted glass and concrete which have high reflectivity and small transitivity, with reflection coefficients of 0.896 , and $40.1 \mathrm{~dB}$ penetration loss. In addition, indoor 
materials have lower penetration loss: $6.84 \mathrm{~dB}$ for indoor walls, $2.9 \mathrm{~dB}$ for clear glass, and $28.3 \mathrm{~dB}$ for brick pillars. Their data shows that indoor to outdoor propagation is difficult at $28 \mathrm{GHz}$. In the same paper, they also presented $28 \mathrm{GHz}$ Urban propagation measurements in New York City. For large scale parameters, best signal level LOS PLE of $n=1.68$ was obtained. For all LOS data acquired, the LOS PLE is $n=2.55$. The PLE for the best NLOS cases are $n=4.58$, and average PLE of $n=5.76$ for all NLOS cases.

S. Sun et al. from the Rappaport research group, presents her work on small scale fading study at $73 \mathrm{GHz}$ in urban environments [11]. They used a channel sounder with a linear track and pan-tilt unit to move a receiver up to 78 wavelength in steps of $\lambda / 2(2.04 \mathrm{~mm})$ and 360 degree sweep in the azimuth plane. Her work uses a statistical approach, shows the omnidirectional small-scale spatial fading and spatial autocorrelation, and directional small-scale spatial fading and spatial autocorrelation. For an omnidirectional model, the LOS fading obeys Ricean distribution with a Kfactor of $10 \mathrm{~dB}$, and NLOS fading was described by log-normal distribution with $\sigma$ of $0.65 \mathrm{~dB}$. For a directional antenna pattern, fading for both LOS and NLOS environments follows Ricean distribution. The K-factor ranges from 7 to $17 \mathrm{~dB}$ for LOS, and 9 to $21 \mathrm{~dB}$ for NLOS. They also show the spatial autocorrelation of received signal amplitude follows the sinusoidal-exponential distribution in LOS environments for omnidirectional antenna patterns, and most directional antenna patterns, and exponential distribution for directional antenna patterns with stepping RX locations parallel to T-R line.

D. Matolak et al. from the University of South Carolina, presented their large scale measurement at 5 and $31 \mathrm{GHz}$ at the Jim Hamilton L. B. Owens Airport [12]. They found the channel at the airport terminal building is close to indoor office 
environments: LOS PLE are close to $n=2$ and NLOS PLE are around $3-4$, with larger standard deviations. In the hangar environment, for both frequency bands, PLE are around $n=3$, with small $\sigma, 2.8 \mathrm{~dB}$ for $5 \mathrm{GHz}$ and $6.3 \mathrm{~dB}$ for $31 \mathrm{GHz}$. The $\sigma$ obtained is smaller than expected as it is from a rich scattering environment.

\subsection{Contribution and structure of the thesis}

In this thesis, we present extensive channel measurement at 73 and $81 \mathrm{GHz}$ at multiple environments. A large scale measurement campaign was carried out at the Boise Airport terminal and tarmac. We analyzed the close-in reference model and floatingintercept models. In addition, our collaboration work with the University of South Carolina and North Carolina State University is presented. We performed attenuation measurements for common building materials for both wideband and narrowband signals at 28(NCSU), 73(BSU), and 91(USC) GHz. Furthermore, this thesis includes indoor and outdoor $73 \mathrm{GHz}$ and $81 \mathrm{GHz}$ small scale fading measurements at BSU and the Boise airport.

The rest of the thesis is structured as follows, Chapter 2 discusses radio wave propagation and channel models. Chapter 3 presents the measurement campaigns, which consist of hardware, environment, procedure and results. Chapter 4 concludes the thesis. 


\section{CHAPTER 2}

\section{OVERVIEW OF CHANNEL MODELING}

Wireless radio channels as a medium between transmitters and the receivers places a challenge in wireless communication systems. It effects the transmitter power requirements, antenna requirements, interference levels, etc. The transmission path between the transmitter and the receiver can vary from simple line-of-sight to one that is obstructed by buildings and other objects. The obstacles can change over time, which makes the wireless channel extremely unpredictable. Other than the obstacles, the motion of the transmitter or the receiver also impacts the signal level. Wireless channel modeling has been one of the most complicated parts of mobile system design.

This chapter provides an overview of radio wave propagation and wireless channel modeling.

\subsection{Radio Wave Propagation}

Electromagnetic waves are reflected, scattered, and diffracted by walls, buildings and other objects while propagating. For mmWave, where the wavelength are smaller than a centimeter, lampposts, human bodies, and trees, are large relative to the wavelength and can cause signal blockage (e.g. shadowing) when an relative large obstacle is in the way of the LOS path between transmitter and receiver. However, the signal still travels from transmitter to receiver by reflecting and scattering. In 
the rest of this section, we will discuss diffraction, reflection, and scattering as they are the basic radio wave propagation mechanisms.

Diffraction is one of the mechanisms that supports radio communication when an obstruction is blocking the TX-RX path. Diffraction occurs when the obstruction has sharp edges. The obstructing surface results in the wave being "bent" around the edge. Thus the wave could travel from transmitter to receiver even without a LOS path. While diffraction is powerful with sub-6 GHz cellular systems, diffraction on mmWave bands becomes unreliable. Due to the small wavelength with mmWave, diffraction is the weakest and least reliable mechanism, whereas scattering and reflection will become more dominant [3].

Reflections occur when the wave collides into a relative large surface. The reflection also depends on the frequency, dielectric constant, permeability, and conductivity of the surface, and the incident angle of the electromagnetic wave. Recent work at $28 \mathrm{GHz}$ shows that outdoor building materials are very reflective $[6,7]$. These works show that mmWave multipath signals can propagate by bouncing off objects, instead of penetrating, which means multipath components can be used for mmWave to establish a viable connection.

Scattering occurs when the wave impinges on a rough surface with the reflected energy spread into all directions. For practice, small objects such as street signs and foliage include scattering in radio paths.

\section{$2.2 \quad$ Propagation Modeling}

Channel models can be used to estimate the potential system capacity and outage for designing a radio system [13]. MmWave frequencies are affected by large scale 
path loss from electromagnetic wave propagation and antenna gain(as in today's sub-6GHz frequency). Path loss for mmWave is severely more than the sub-6 GHz frequency from atmospheric by molecular resonance, rain and fog, due to the small wavelength [3]. Other than path loss, mmWave links also analyzed its received signal fluctuation due to the environment, this effect is a small-scale channel effect. In the rest of this section we are going to discuss channel models and parameters for large scale and small scale channel effects.

\subsubsection{Large scale propagation channel effects}

\section{Free Space model}

In large scale propagation channel effects, the free space propagation model is often used as a starting point for the evaluation of the radio system. The free space propagation model considers that the electromagnetic wave is in free space, with no obstacles, no reflection, and no scattering. Harold T. Friis in 1946 [14] introduced the free space equation,

$$
P_{r}(d)=\frac{P_{t} G_{t} G_{r} \lambda^{2}}{(4 \pi)^{2} d^{2}}
$$

where,

$P_{r}(d)$ - received power,

$d$ - TX-RX separation,

$P_{t}$ - transmitted power,

$G_{t}$ - transmitter antenna gain,

$G_{r}$ - receiver antenna gain,

$\lambda$ - wavelength.

The Friis free space equation (2.1) implies the received power falls off as the square 
of the TX-RX separation, which means the power decays with a distance at $20 \mathrm{~dB}$ /decade.

The path loss, including the effect of antenna gain, which is the attenuation (dB), can be expressed as,

$$
P L(d B)=10 \log \frac{P_{t}}{P_{r}}=-10 \log \left[\frac{G_{t} G_{r} \lambda^{2}}{(4 \pi)^{2} d^{2}}\right]
$$

Or as,

$$
P L(d)=P_{t}[d B m]-P_{r}(d)[d B m]+G_{t}[d B]+G_{r}[d B]
$$

where,

$P_{r}(d)$ - received power at distant $\mathrm{d}$,

$d$ - TX-RX separation,

$P_{t}$ - transmitted power,

$P L$ - Path loss at distant $\mathrm{d}$,

$G_{t}$ - transmitter antenna gain in decibel,

$G_{r}$ - receiver antenna gain in decibel.

We used this model for penetration loss measurement at 73 and $81 \mathrm{GHz}$ [15], where the attenuation is calculated as the difference of path loss. We will further discuss this is section 4 .

\section{Log-distance model}

As the measurement and FSPL both show that the path loss is proportional to distance logarithmically, the following model is used to describe the average large-scale path loss $P L$ for changing distant $d$, by using a path loss exponent(PLE) $n$ 


$$
\overline{P L}(d) \propto\left(\frac{d}{d_{0}}\right)^{n}
$$

or

$$
\overline{P L}(d B)=\overline{P L}\left(d_{0}\right)+10 n \log \left(\frac{d}{d_{0}}\right)
$$

where $n$ is the path loss exponent which indicate the rate of path loss increases with distant $d, d_{0}$ is the close-in measurement close to the transmitter [16]. This model requires close-in measurement in the environment.

\section{Large-Scale Path Loss Model for Outdoors Channels}

There are many models developed to characterize large scale path loss at different frequency and in different outdoor environments. Following are some commonly used models:

The Long-Rice model is used to predict path loss for point-to-point communication systems from $20 \mathrm{MHz}$ to $20 \mathrm{GHz}$, over different kinds of terrain (e.g. for different ground refractivity, ground conductivity) and weather. Many applications (e.g.Nautel and QRadioPredict) use this model.

The Okumura model is wildly used in urban areas for frequency ranges from 150 to $1920 \mathrm{MHz}$ and 1 to $100 \mathrm{~km}$ separation. The path loss is computed with FSPL, median attenuation, antenna gain, and environment gain.

The Hata model is developed as an extension of the Okumura model with a correction factor applied. The Hata model is developed for urban, suburban and open environments.

In our study [17], we used the close-in free space reference distance (CI) model and floating intercept (FI) model. These models are used for mmWave channel measure- 
ment in recent studies $[8,9,18]$. The Close-in free space reference distance model, also known as the Log-normal shadowing model, is developed based on the log-distance model (2.5). Over numbers of measurement with the same TX-RX separation, each path might face different obstacles causing different amounts of shadowing effect to the received signal. This phenomenon is called log-normal shadowing. In this model, random variable $X_{\sigma}$ is added for randomness brought by shadowing effects,

$$
P L(d)[d B]=\overline{P L}(d)+X_{\sigma}=\overline{P L}\left(d_{0}\right)+10 n \log _{10}\left(\frac{d}{d_{0}}\right)+X_{\sigma}
$$

Where $d_{0}$ is the close-in free space reference distant, $d$ is the TX-RX separation, $n$ is the path loss exponent. $X_{\sigma}$ is a zero-mean Gaussian random variable with standard deviation $\sigma$, both $X_{\sigma}$ and $\sigma$ are in $\mathrm{dB} . \overline{P L}\left(d_{0}\right)$ is the close-in free space path loss in $\mathrm{dB}$ that is given by,

$$
\overline{P L}\left(d_{0}\right)=20 \log _{10} \frac{4 \pi d_{0}}{\lambda}
$$

The Floating intercept model (2.8), introduced in [19], has no physical reference, but fits the best line to the measurement data with least square regression. The FI model is only valid over the specific distances for which measurement was made.

$$
P L(d)[d B]=\alpha+10 \beta \log _{10}(d)+X_{\sigma}
$$

where $\alpha$ is a floating intercept in $\mathrm{dB}, \beta$ is the linear slope, and $X_{\sigma}$ is zero-mean random variable with standard deviation $\sigma$ in $\mathrm{dB}$ to model shadow fading. $\beta$ is similar to the PLE in CI model, but $\beta$ only serves as the slope for the linear line for best fit of scattered data, but without physical basis or frequency dependence [19]. 


\subsubsection{Small scale propagation channel effects}

Small scale fading is used to describe the rapid variations of the amplitude, phase, or propagation delays from multipaths, over a period of time or distance. Small scale fading is caused by interference from multiple versions of transmitted signal arriving at the receiver at slightly different times. These different transmitted signals or waves are multipath waves. Multipath waves arrive the receiver at slightly different times with different amplitude and phase.

Small scale fading can cause rapid changes in signal strength, frequency modulation, and time dispersion, which places limitations on system design in physical layer. Many physical factors can cause these channel effects. In [16], the author summarized the factors as following:

- Multipath Propagation

- Speed of the model

- Speed of surrounding objects

- The transmission bandwidth of the signal 


\section{CHAPTER 3}

\section{MEASUREMENT CAMPAIGN AND ANALYSIS}

\subsection{Large Scale fading Measurement at Boise Airport}

During the summer of 2018 and 2019, we performed several channel measurement campaigns at the Boise airport in both indoor and outdoor environments for large scale fading. In this section, we are presenting the work at the airport.

\subsubsection{System setup}

Table 3.1: Hardware Specifications of 73 and $81 \mathrm{GHz}$ Channel Measurements [1]

\begin{tabular}{|c||c|c|}
\hline \multicolumn{1}{|c||}{ Specifications } & $\mathbf{7 3 ~ G H z}$ & $\mathbf{8 1 ~ G H z}$ \\
\hline \hline Center frequency & $73 \mathrm{GHz}$ & $81 \mathrm{GHz}$ \\
\hline IF frequency & $4 \mathrm{GHz}$ & $3 \mathrm{GHz}$ \\
\hline LO frequency & $2 \times 38.5 \mathrm{GHz}$ & $2 \times 39 \mathrm{GHz}$ \\
\hline Modulation scheme & BPSK & BPSK \\
\hline Bandwidth & $1.3 \mathrm{GHz}$ & $1.3 \mathrm{GHz}$ \\
\hline TX and RX antenna Type & \multicolumn{2}{|c|}{$\mathrm{Horn}$ Antenna } \\
\hline TX antenna gain & $24 \mathrm{dBi}$ & $24 \mathrm{dBi}$ \\
\hline TX azimuth antenna HPBW & $7 \circ$ & $7 \circ$ \\
\hline TX elevation antenna HPBW & $11 \circ$ & $11 \circ$ \\
\hline RX antenna gain & $24 \mathrm{dBi}$ & $24 \mathrm{dBi}$ \\
\hline RX azimuth antenna HPBW & $7 \circ$ & $7 \circ$ \\
\hline RX elevation antenna HPBW & $11 \circ$ & $11 \circ$ \\
\hline Antenna polarization (TX-to-RX) & $\mathrm{V}-\mathrm{V}$ & $\mathrm{V}-\mathrm{V}$ \\
\hline Max transmit power & $6 \mathrm{dBm}$ & $3 \mathrm{dBm}$ \\
\hline
\end{tabular}


For all of our measurement at 73 and $81 \mathrm{Ghz}$, the system setup is similar to a heterodyne system. On the transmitter side, shown in fig. 3.1, Keysight M8190A Arbitrary waveform generator (AWG) outputs a wideband signal with center frequency at 3 or $4 \mathrm{GHz}$, then the waveform is up converted with an VDI WR12CCU up-converter with LO signal generated from a Keysight N5183B signal generator. The RF signal out of the up-converter is pushed through a filter and $20 \mathrm{~dB}$ gain power amplifier(PA) then transmitted with a directional horn antenna with $24 \mathrm{dBi}$ gain and beamwidth of $11^{\circ}$ and $7^{\circ}$ in the azimuth and the elevation angles. On the receiver side, shown in fig. 3.2, the RF signal goes through the antenna(same as TX), filter, and a $20 \mathrm{~dB}$ gain LNA, then to the VDI WR12CCD down-converter, with LO signal from another signal generator(same as TX). The output IF signal from the down-converter is analyzed with a Keysight DSAV084A Oscilloscope and 89600 VSA software. A detailed hardware description is in Table 3.1.

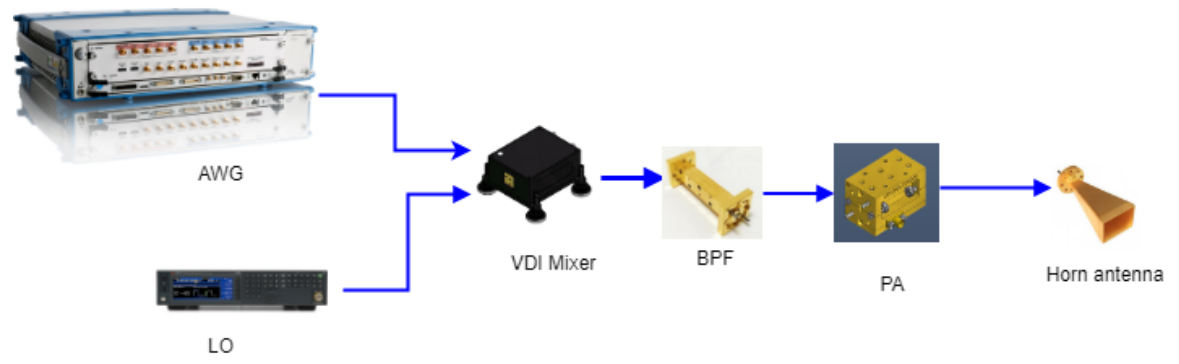

Figure 3.1: System diagram of transmitter [1]

\subsubsection{Measurement Environment}

The indoor and outdoor large scale path loss measurement took place at the Boise airport, in terminal concourse $\mathrm{C}$ and on the tarmac between the $\mathrm{B}$ gates and $\mathrm{C}$ gates. 


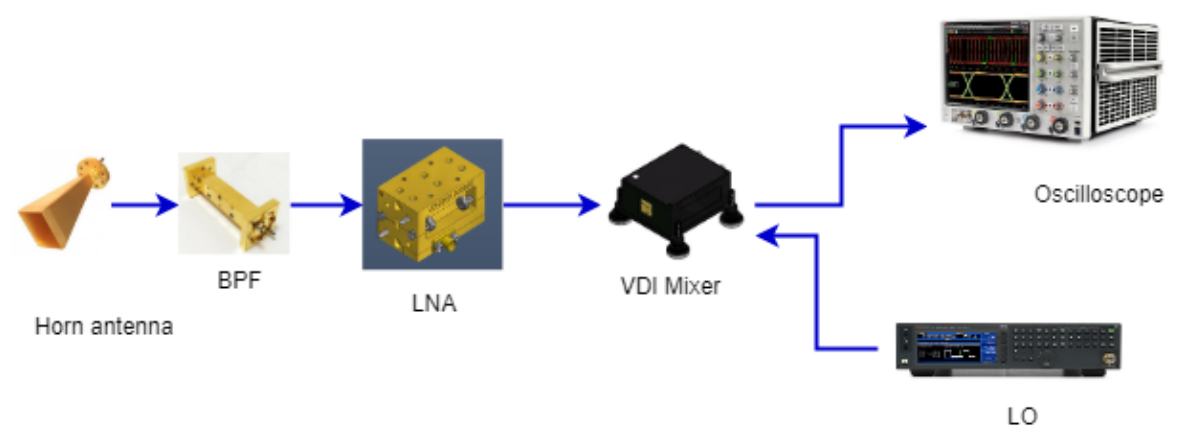

Figure 3.2: System diagram of receiver [1]

Fig.3.3 shows the floor plan of the concourse $\mathrm{C}$ area, a typical concourse area for a small airport. There are 10 gates, a set of escalators, a gift shop, a coffee shop, restrooms, a couple of pillars, a seating area with 250 seats, and a office style cubical area. In concourse $\mathrm{C}$, the carpet floor covers the cubical area and the seating area on the west and south side, the rest floor is made of concrete. Above the carpet, the ceilings are fiber tiles. Above the concrete, the ceilings are metal with little holes for noise cancellation. The south side of the concourse $\mathrm{C}$ area are gates, and clear glass is used as walls, the rest of the walls in the area are drywall. The transmitter was placed against the north side wall, next to several chairs and desks. The receiver was moving towards the cubical area, seating area, around pillars and escalators.

The outdoor measurement took place outside on the tarmac between gate B and $\mathrm{C}$, as shown in fig.3.4. The transmitter was placed next to the boarding bridge and terminal building. During the measurement there can be aircraft and pickup trucks in the surrounding area. RX moves along the tarmac in different directions as labeled by the red dotted line. NLOS environment was created by placing RX behind the truck, minivan, and other obstacles. 


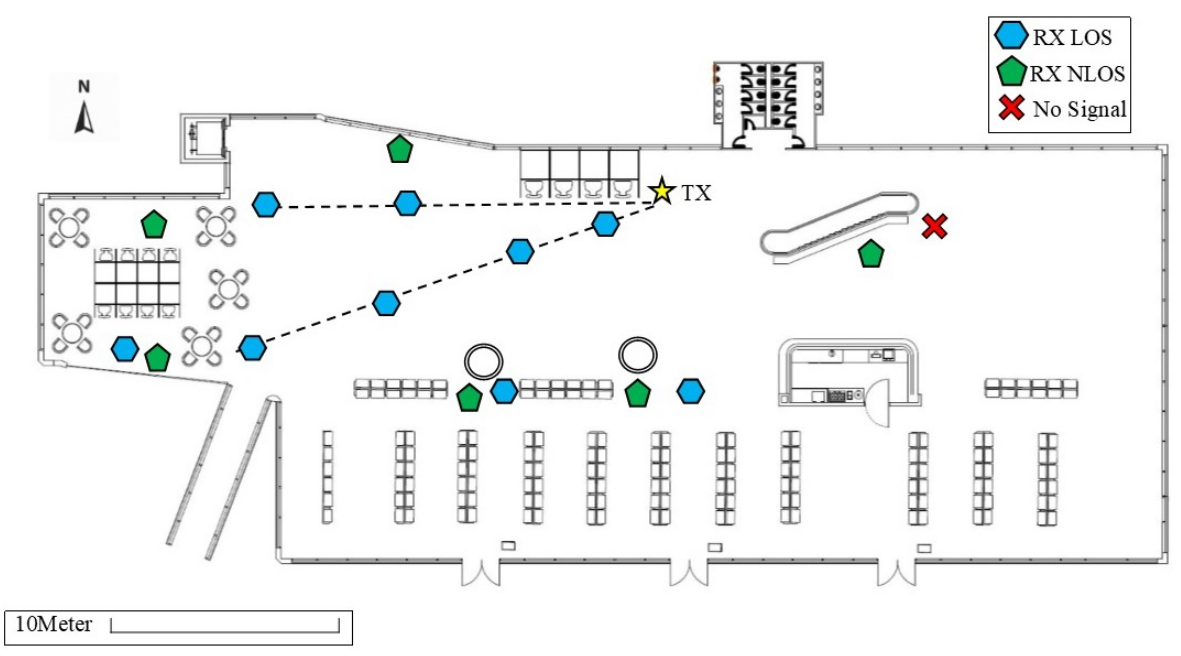

Figure 3.3: Layout of concourse $\mathrm{C}$ area at Boise Airport, with LOS and NLOS TX RX locations [1]

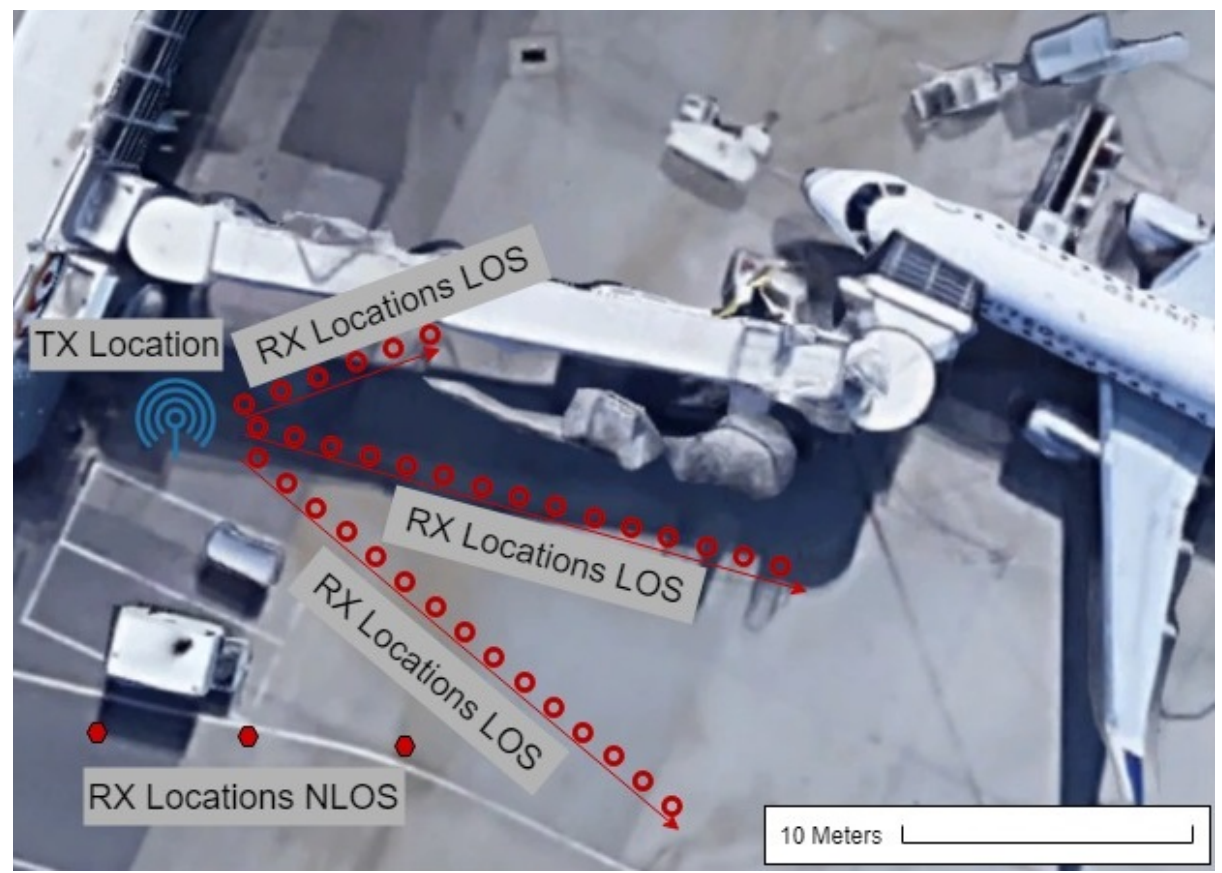

Figure 3.4: Map of tarmac at Boise Airport, showing TX and RX locations [1]

\subsubsection{Measurement Procedure}

During the measurement, TX was placed in one position with six different RX locations for both LOS and NLOS. The Maximum distance measured between TX 
and RX is $22 \mathrm{~m}$ indoor and $20 \mathrm{~m}$ outdoor. The distance was measured with laser measurement for accuracy. The height of the TX and RX antennas indoors are 1.3 $\mathrm{m}$ and $1.2 \mathrm{~m}$, and $1.5 \mathrm{~m}$ and $1.2 \mathrm{~m}$ outdoor.

At each RX location, the receiver was mounted to a linear rail and moves to 5 different positions with half wavelength separations, which is $2.05 \mathrm{~mm}$ for $73 \mathrm{GHz}$ measurements and $1.86 \mathrm{~mm}$ for $81 \mathrm{GHz}$ measurements. More about the linear rail system could be found in Appendix A. At each linear rail position, a power delay profile (PDP) is captured through VSA software and stored in an excel file on a laptop. Signals at some of the NLOS locations are not detectable.

\subsubsection{Data Analysis and Result}

Large scale path loss is calculated based on the PDP captured from the measurement. Due to the noise in the captured PDPs, we estimate the noise and selected a threshold level that is $15 \mathrm{~dB}$ lower than the highest peak in PDP. Thus, the noise is removed, and the rest of the multi-path components are added as the received power calculation. In [20], they describe the received power as,

$$
P_{r}=P_{0} \sum_{L}^{i=1}\left|\beta_{i}\right|^{2}
$$

where $L$ is the number of the multipath and $\beta$ is the amplitude of the multipath.

$\mathrm{CI}$ and FI models are used to estimate the large scale fading parameters, as we discussed in section 2.2.1. Fig. 3.5 shows the measured data and $\operatorname{CI}\left(d_{0}=1 \mathrm{~m}\right)$ and FI models for $73 \mathrm{GHz}$ and $81 \mathrm{GHz}$ measurements for the Boise airport indoor environment. Fig. 3.6 shows the measured data and $\mathrm{CI}\left(d_{0}=1 \mathrm{~m}\right)$ and FI models for $73 \mathrm{GHz}$ and $81 \mathrm{GHz}$ measurements for the Boise airport outdoor environment. The 
FSPL line provides a physically based reference point for estimating LOS path loss, as both models are close to FSPL $n=2$ line.

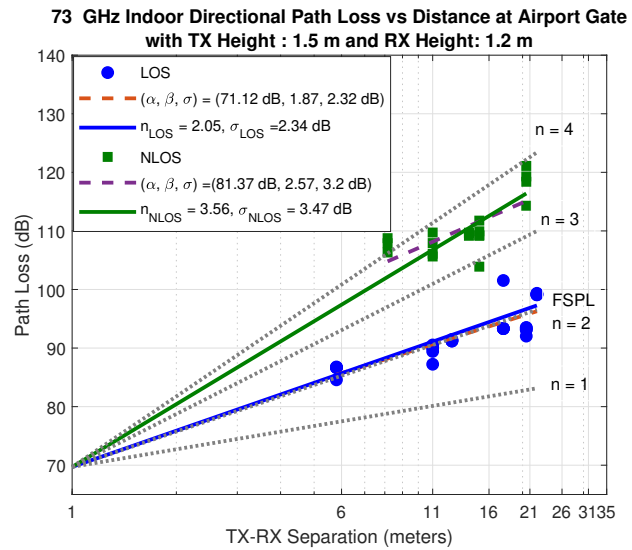

(a) $73 \mathrm{GHz}$ indoor directional path loss

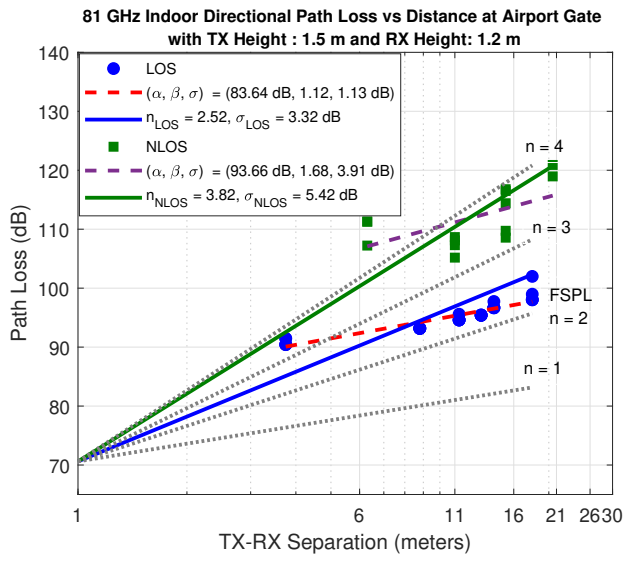

(b) $81 \mathrm{GHz}$ indoor directional path loss

Figure 3.5: $73 \mathrm{GHz}$ and $81 \mathrm{GHz}$ indoor directional CI $\left(d_{0}=1\right)$ and FI path loss models with TX antenna heights of $1.5 \mathrm{~m}$ and RX antenna heights of $1.2 \mathrm{~m}$ at Boise Airport concourse C. Blue dots are LOS path loss values, green squares are obstructed NLOS path loss values. [1]

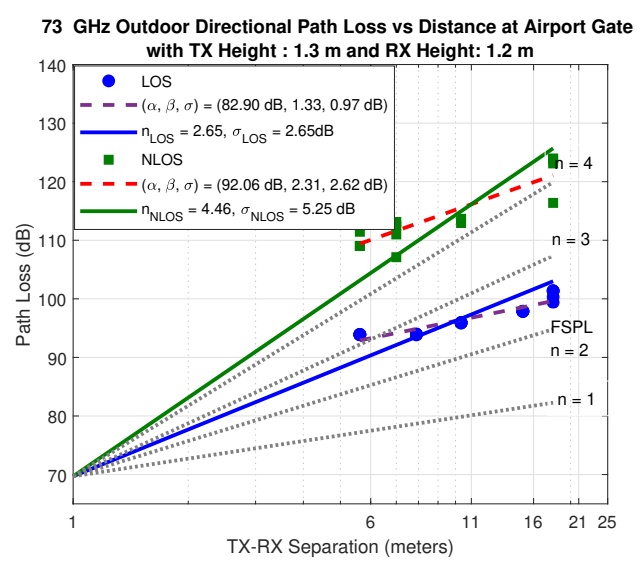

(a) $73 \mathrm{GHz}$ outdoor directional path loss

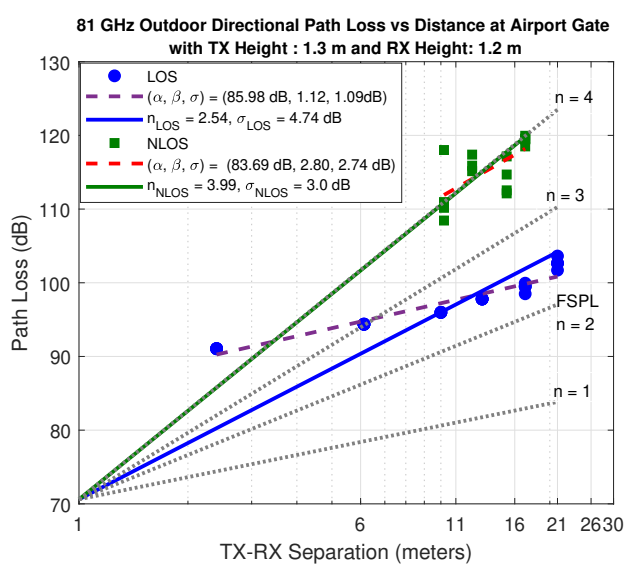

(b) $81 \mathrm{GHz}$ outdoor directional path loss

Figure 3.6: $73 \mathrm{GHz}$ and $81 \mathrm{GHz}$ outdoor directional CI $\left(d_{0}=1\right)$ and FI path loss models with TX antenna heights of $1.3 \mathrm{~m}$ and RX antenna heights of $1.2 \mathrm{~m}$ at Boise Airport tarmac. Blue dots are LOS path loss values, green squares are obstructed NLOS path loss values. [1] 


\section{Close-in free space reference distance path loss model}

Recall equation (2.6), PLE $n$ is computed with best fit MMSE over all measurements from this campaign. $d_{0}=1 m$ is used in several different works $[9,21]$, to simplify the modeling and comparison of measurements.

Table 3.2: Directional close-in free space reference distance $\left(d_{0}=1 \mathrm{~m}\right)$ path loss models EQ.(2.6) for LOS and NLOS. $n$ is the PLE and $\sigma$ is the standard deviation of the shadow factor(a zero mean random Gaussian variable) [1]

\begin{tabular}{|c|c|c|c|c|c|}
\hline \multicolumn{5}{|c|}{ Directional CI path loss with $d_{0}=1 m$} \\
\hline \multirow{2}{*}{ Frequency } & \multirow{2}{*}{ Environment } & \multicolumn{2}{|c|}{ LOS } & \multicolumn{2}{c|}{ NLOS } \\
\cline { 3 - 6 } & & $\mathrm{n}$ & $\sigma, \mathrm{dB}$ & $\mathrm{n}$ & $\sigma, \mathrm{dB}$ \\
\hline \multirow{2}{*}{$73 \mathrm{GHz}$} & Indoor & 2.05 & 2.34 & 3.56 & 3.47 \\
\cline { 2 - 6 } & Outdoor & 2.65 & 2.65 & 4.46 & 5.25 \\
\hline \multirow{2}{*}{$81 \mathrm{GHz}$} & Indoor & 2.52 & 3.32 & 3.82 & 5.42 \\
\cline { 2 - 6 } & Outdoor & 2.54 & 4.74 & 3.99 & 3.00 \\
\hline
\end{tabular}

Table 3.2 provides the CI model parameters with $d_{0}=1 \mathrm{~m}$, for both indoor and outdoor environments and LOS and NLOS cases at 73 and $81 \mathrm{GHz}$ mmWave bands. The $73 \mathrm{GHz}$ LOS PLEs for indoor and outdoor environment are 2.05 and 2.65, which is comparable to FSPL of $n=2$ (20 dB attenuation per decade of TX-RX separation). The standard deviations of shadow factor at $73 \mathrm{GHz}$ for the indoor environment are $2.34 \mathrm{~dB}$ and $2.65 \mathrm{~dB}$. The $81 \mathrm{GHz}$ LOS PLEs are 2.52 and 2.54 for indoor and outdoor cases, which the indoor PLE is slightly higher than $71 \mathrm{GHz}$. Also, the LOS shadow factor standard deviation at $81 \mathrm{Ghz}$ is $3.32 \mathrm{~dB}$ and $4.47 \mathrm{~dB}$, which is also higher than $71 \mathrm{GHz}$. This can be attributed to the difficulty in aligning the narrow beam directional antennas. As the LOS PLEs is $n=2.5$ for both bands, this indicates the impact of beam pointing in future mmWave wireless systems. To improve the LOS field measurement, a laser meter is used to ensure alignment. For NLOS scenarios, both bands in both environments have higher PLE and shadow factor 
standard deviation. It indicates a larger rate of path loss increases with distance and more fluctuations in received signal strength. This is most likely due to the obstacles around the environment.

\section{Floating intercept path loss model}

Table 3.3 provides the FI model parameters. The $\alpha$ intercept for $73 \mathrm{GHz}$ LOS are $71.12 \mathrm{~dB}$ at indoor and $82.90 \mathrm{~dB}$ at outdoor, which is close to $73 \mathrm{GHz} 1 \mathrm{~m} \mathrm{FSPL}$ value(used in CI model), $69.7 \mathrm{~dB} . \alpha$ is $83.64 \mathrm{~dB}$ and $85.98 \mathrm{~dB}$ for $83 \mathrm{GHz}$ indoor and outdoor LOS scenarios, as the $1 \mathrm{~m}$ FSPL at $81 \mathrm{GHz}$ is $70.6 \mathrm{~dB}$. Also, we are seeing the $\beta$, the slope of the fit line for all cases are lower than the CI model, so is the SF standard deviation. This is because the FI model finds a slightly better fit to measured data without the constraint and usually results in a lower standard deviation. T.S. Rappaport, et al. in their study [9], indicate that the FI model is very sensitive with post processing. They also proposed to use the CI model with a $1 \mathrm{~m}$ reference distance due to its physical basis (in the FI model $\alpha$, and $\beta$ have no physical meaning), stability, and benefit with the standard model to compare with different measurements.

Table 3.3: Directional floating intercept path loss models for LOS and NLOS. $\alpha$ is the floating intercept, $\beta$ is the slope of the MMSE fitted line and $\sigma$ is the standard deviation of the shadow factor [1]

\begin{tabular}{|c|c|c|c|c|c|c|c|}
\hline \multicolumn{9}{|c|}{ Directional FI path loss model } \\
\hline \multirow{2}{*}{ Frequency } & \multirow{2}{*}{ Environment } & \multicolumn{3}{|c|}{ LOS } & \multicolumn{3}{c|}{ NLOS } \\
\cline { 3 - 8 } & & $\alpha, \mathrm{dB}$ & $\beta$ & $\sigma, \mathrm{dB}$ & $\alpha, \mathrm{dB}$ & $\beta$ & $\sigma, \mathrm{dB}$ \\
\hline \multirow{2}{*}{$73 \mathrm{GHz}$} & Indoor & 71.12 & 1.87 & 2.32 & 81.37 & 2.57 & 3.20 \\
\cline { 2 - 8 } & Outdoor & 82.90 & 1.33 & 0.97 & 92.06 & 2.31 & 2.62 \\
\hline \multirow{2}{*}{$81 \mathrm{GHz}$} & Indoor & 83.64 & 1.12 & 1.13 & 93.66 & 1.68 & 3.91 \\
\cline { 2 - 8 } & Outdoor & 85.98 & 1.12 & 1.09 & 83.69 & 2.80 & 2.74 \\
\hline
\end{tabular}




\subsection{Attenuation of Different Building Materials}

This section presents the collaboration work between the University of South Carolina, at $91 \mathrm{GHz}$, Boise State University at $73 \mathrm{GHz}$, and North Carolina State University at $28 \mathrm{GHz}$, mainly our work at BSU.

\subsubsection{System setup}

The system setup for this work is close to the setup we presented in section 3.1.1, with different antennas and without linear rail. Due to some of the requirements of the measurement, a small dimension antenna is used on the TX and a WR-12 waveguide input port on the PA as an RF input on the RX side. Table 3.4 shows the hardware specification we used in the measurement.

Table 3.4: Hardware Specifications for $73 \mathrm{GHz}$ Measurement

\begin{tabular}{|c|c|}
\hline Center Frequency $(\mathrm{GHz})$ & $73 \mathrm{GHz}$ \\
\hline Wave Length $(\mathrm{mm})$ & 4.1 \\
\hline Wideband Signal & Radio Chirp \\
\hline Bandwidth $(\mathrm{MHz})$ & 500 \\
\hline Narrowband Signal & CW \\
\hline TX Antenna & 12 \\
\hline TX Antenna Gain $(\mathrm{dBi})$ & Vertical-Vertical \\
\hline Antenna Polarization & 1.08 \\
\hline TX Antenna Beam Width $(\mathrm{deg})$ & 5.6 \\
\hline TX Antenna Diameter, A $(\mathrm{cm})$ & 24.8 \\
\hline TX Antenna far-field for dmax $(\mathrm{cm})$ & WR-12 Waveguide \\
\hline Projection Diameter, D for dmax $(\mathrm{cm})$ & \\
\hline RX Antenna & \\
\hline
\end{tabular}

For wideband measurement, on the TX side, AWG outputs a $4 \mathrm{GHz}$ IF signal with $500 \mathrm{MHz}$ bandwidth. The IF signal feeds into an up-converter, with another LO input at $38.5 \mathrm{GHz}$ (multiplied by two in the up-converter) outputs a $73 \mathrm{GHz}$ signal. 
The RF signal is transmitted through a bandpass filter, PA, then a horn antenna. On the RX side, a bandpass filter with a WR-12 waveguide is used to capture the RF signal, then through an LNA, the signal is fed into a down-converter. With LO at $38.5 \mathrm{GHz}$, the IF received signal is at $4 \mathrm{GHz}$. The scope and VSA software is used to analyze the IF signal. An SMA cable with IF signal connects AWG and the scope for signal correlation in time domain. For narrow band measurement, a CW signal is used for measurement.

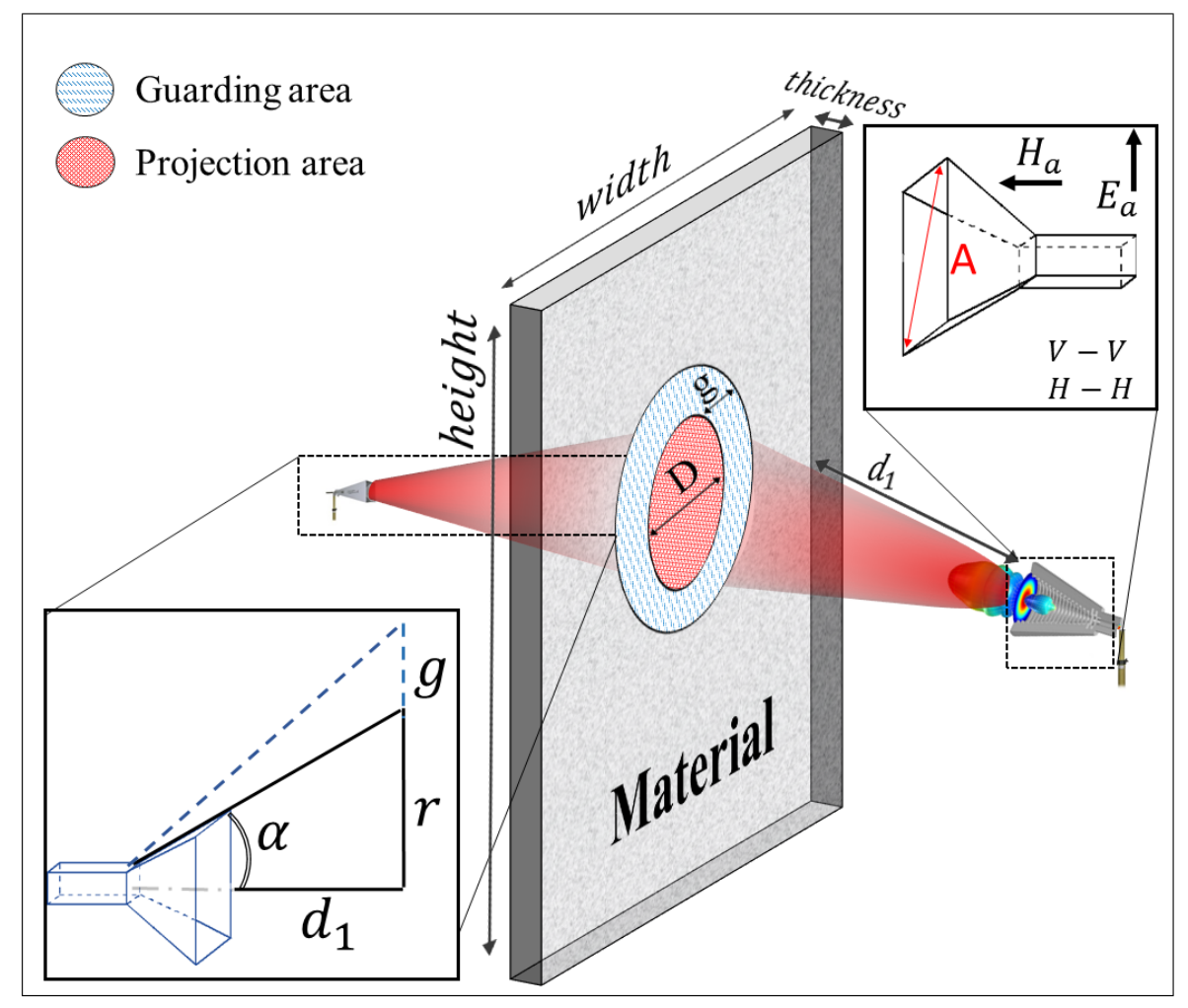

Figure 3.7: Geometry for determining projection area and material dimensions with antenna dimension [2]. 


\subsubsection{Measurement Procedure}

In this work, plywood, acoustic ceiling, clear glass, drywall, and cinder blocks are used for the measurement. To minimize the variation from materials, three universities purchased the same brand from the same vendor across the US. Plywood is composited with several layers of wood glued together in a perpendicular direction. It is widely used for residential light construction. Drywall is made from gypsum plaster between two sheets of thick paper. It is commonly used for interior walls. We used common mineral fiber ceiling tiles. The cinder blocks we used in this measurement have two holes, which means throughout the incident surface, the material is inhomogeneous. Glass is also a commonly used material in modern buildings.

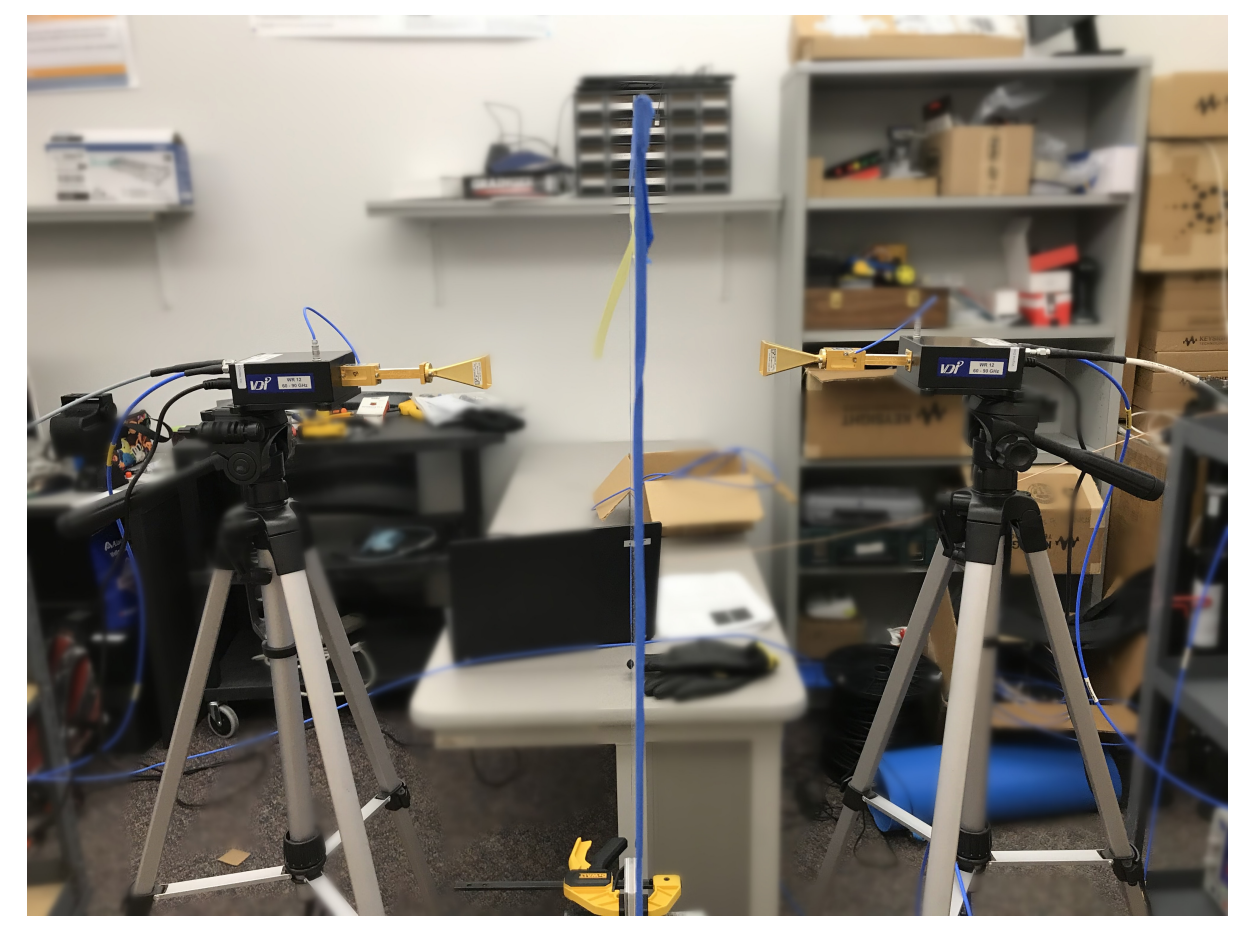

Figure 3.8: Measurement picture for glass, with RX on the left and TX on the right. In the measurement a smaller dimension antenna was used for TX and waveguide was used for RX.

The materials are large enough to cover the projection area of the main lobe of the 
antenna. Fig. 3.7 shows the projection dimension for the antenna, where projection diameter is $\mathrm{D}$,

$$
D=2 d * \tan (\alpha)
$$

where $\alpha$ is half antenna beamwidth and $d$ is the separation between antenna and the material. The guard projections width $g=r / 2$, where $r$ is the radius of the projection area or $r=D / 2$. All the materials in this work were chosen with greater dimensions than the projection and the guard annulus. Fig. 3.8 shows the testing setup for clear glass measurement, note that during the actual measurement, a smaller dimension antenna was used for TX, and a waveguide was used for RX to meet the far-field requirement.

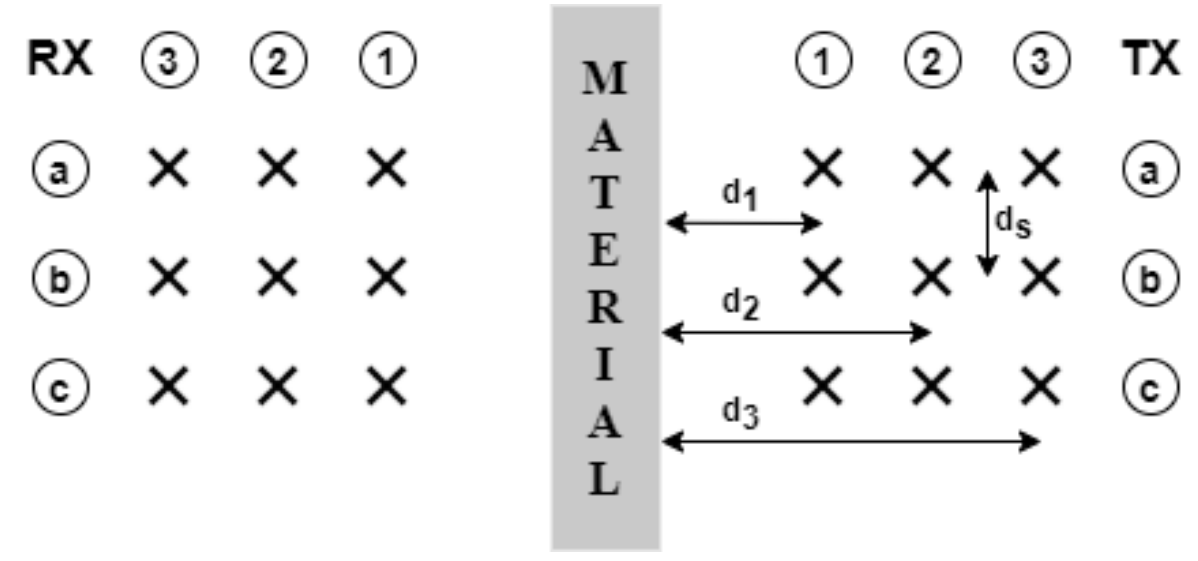

Figure 3.9: Measurement diagram, top view, with material in the middle, RX locations on the left, and TX locations on the right.

The measurement was conducted in the Signal Processing and Wireless Communication lab at BSU. TX and RX antennas were placed at far field distances where far field is, 


$$
d>2 A^{2} / \lambda
$$

where $d$ is the TX-RX separation, $\lambda$ is the wavelength, and $A$ is horn antenna aperture dimension.

Fig. 3.9 shows the 9 locations of TX and RX antenna placement, where TX-RX separations of $d_{1}, d_{2}$, and $d_{3}$ are $20,25,30 \mathrm{~cm}$. Distance between two locations at the same TX-RX separation, $d_{s}$, is $4 \mathrm{~cm}$. At each location, TX and RX antenna are placed to face each other and perpendicular to the material as the example from the National Institute of Standards and Technology [22]. Having multiple locations minimizes error from antenna misalignment and material inhomogeneity. Attenuation $L$ was calculated with LOS path loss and path loss attenuated by material as,

$$
L(d B)=P L^{M U T}(d)-P L^{L O S}(d)
$$

where, $P L^{M U T}(d)$ is the path loss with material under test, $P L^{L O S}(d)$ is $\operatorname{LOS}$ unobstructed path loss. The path loss is calculated with (2.3).

Table 3.5: Average specific attenuation with standard deviation at $28 \mathrm{GHz}$ (NCSU), $73 \mathrm{GHz}(\mathrm{BSU})$ and $91 \mathrm{GHz}$ (USC) for wide band measurements. Cinder block attenuation at 91 and $73 \mathrm{GHz}$ is only narrow band result due to large attenuation [2]

\begin{tabular}{|c|c|c|c|c|}
\hline Frequency & Material & Thickness, $\mathrm{mm}$ & Mean attenuation, $\mathrm{dB} / \mathrm{cm}$ & Standard deviation, $\sigma \mathrm{dB} / \mathrm{cm}$ \\
\hline \multirow{4}{*}{$91 \mathrm{GHz}$} & clear glass & 1.8 & 18.79 & 6.39 \\
\cline { 2 - 5 } & plywood & 11.2 & 11.76 & 4.2 \\
\cline { 2 - 5 } & drywall & 13.1 & 1.97 & 1.26 \\
\cline { 2 - 5 } & cinder block & 195 & 2.13 & 0.22 \\
\cline { 2 - 5 } & acoustic ceiling tiles & 11.3 & 1.15 & 1.13 \\
\hline \multirow{4}{*}{$73 \mathrm{GHz}$} & clear glass & 1.8 & 9.07 & 0.55 \\
\cline { 2 - 5 } & plywood & 11.2 & 6.10 & 1.49 \\
\cline { 2 - 5 } & drywall & 13.1 & 1.4 & 0.46 \\
\cline { 2 - 5 } & cinder block & 195 & 2.0 & 0.07 \\
\hline \multirow{5}{*}{$28 \mathrm{GHz}$} & acoustic ceiling tiles & 11.3 & 0.77 & 0.20 \\
\cline { 2 - 5 } & clear glass & 1.8 & 4.38 & 0.18 \\
\cline { 2 - 5 } & plywood & 11.2 & 5.09 & 0.23 \\
\cline { 2 - 5 } & drywall & 13.1 & 0.94 & 0.18 \\
\cline { 2 - 5 } & acoustic ceiling tiles & 11.3 & 1.03 & 0.06 \\
\hline
\end{tabular}




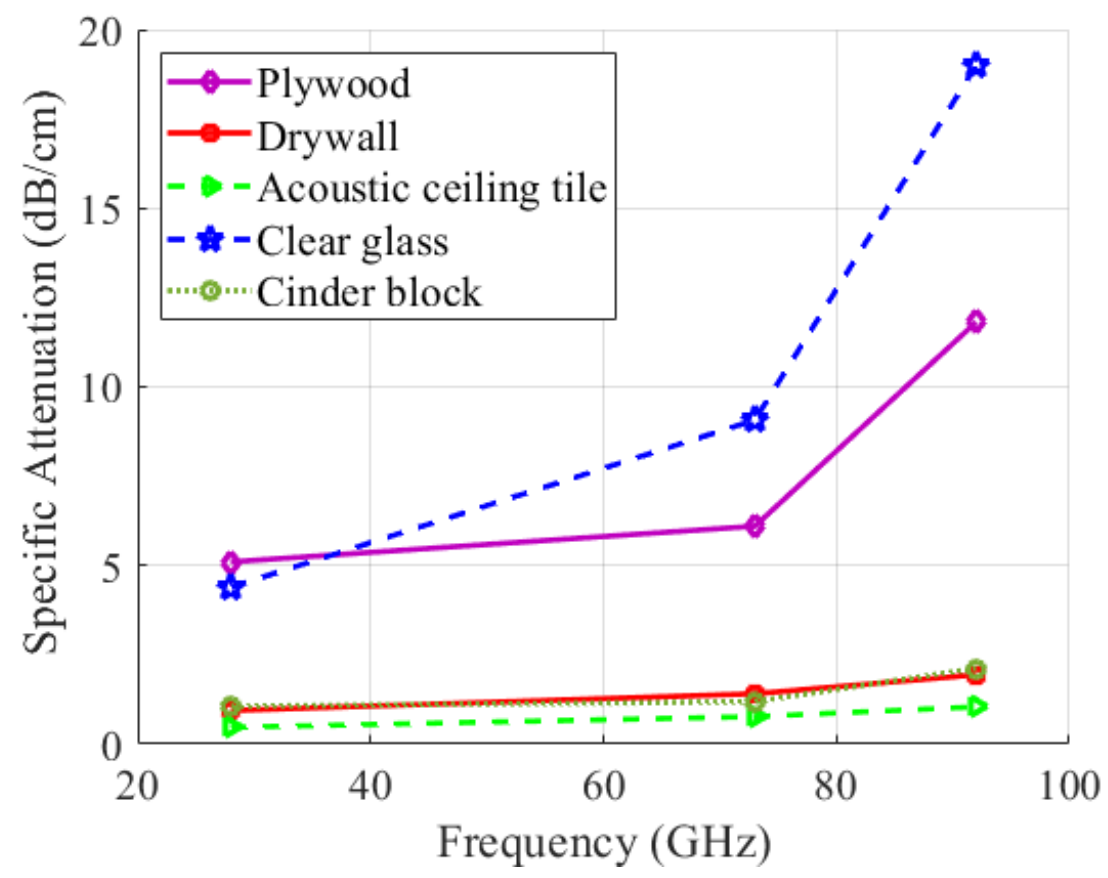

Figure 3.10: Average attenuation versus frequency at 28, 73, $91 \mathrm{GHz}$ for all test materials $[2]$

\subsubsection{Data Analysis Result}

Table 3.5 is from our collaboration work [2]. It presents a summary of average attenuation and standard deviation of measured building materials at 28, 73, and $91 \mathrm{GHz}$ for wideband measurements. At $73 \mathrm{GHz}$, the largest average attenuation is from the clear glass at $9.07 \mathrm{~dB} / \mathrm{cm}$ followed by plywood, cinder block, drywall, and ceiling tiles. The trend we are observing at $73 \mathrm{GHz}$ is the same with $91 \mathrm{GHz}$. At $28 \mathrm{GHz}$, plywood delivers the largest average attenuation at $5.09 \mathrm{~dB} / \mathrm{cm}$, and the smallest average attenuation with ceiling tiles at $0.44 \mathrm{~dB} / \mathrm{cm}$. The trend can be better observed in fig. 3.10.

The average attenuation is obtained as average attenuation over the thickness of the material. Note that the measurement at 73 and $91 \mathrm{GHz}$, due to the thickness of 
the cinder block, the total attenuation is too large for the RX system to detect any in-band signal. Thus, no wideband measurements for the cinder block were obtained. Also, for the wideband measurements, only in-band power is measured. We notice that out of band energy attributes to the small difference, as we compare the wideband and narrow band results at 73 and $91 \mathrm{GHz}$. Detailed figures showing attenuation at different positions can be found in Appendix C.

\subsection{Small scale measurement at Boise State University and Boise Airport}

This sections presents the small scale measurement at $73 \mathrm{GHz}$ and $81 \mathrm{GHz}$ we performed indoors at Boise State University and outdoors at the Boise airport.

\subsubsection{System Setup}

Table 3.6: Hardware Specifications for Small Scale Measurement

\begin{tabular}{|c|c|}
\hline Center Frequency $(\mathrm{GHz})$ & $73 \mathrm{GHz}, 81 \mathrm{GHz}$ \\
\hline LO frequency & $2 \times 38.5 \mathrm{GHz}$ \\
\hline IF frequency & $4 \mathrm{GHz}$ \\
\hline Wideband Signal & Radio Chirp \\
\hline Bandwidth $(\mathrm{MHz})$ & 500 \\
\hline Antenna & 12 \\
\hline Antenna Gain $(\mathrm{dBi})$ & 7 \\
\hline Azimuth antenna HPBW $(\mathrm{deg})$ & 11 \\
\hline Elevation antenna HPBW $(\mathrm{deg})$ & Vertical-Vertical \\
\hline Antenna Polarization & $-5 \mathrm{dBm}$ \\
\hline Max transmit power &
\end{tabular}

The system setup for this work is an extension to the setup we presented in section 3.1.1, with a Pan-Tilt Unit(PTU) added on the TX and RX side for precise antenna 
pointing angles. Detailed specification is in 3.6. Each PTU is controlled by a laptop through a serial cable, and two laptops are connected through UDP. The TX PTU sits on a heavy-duty tripod and carries the up converter. The RX PTU sits on the linear rail, which with steps of $\lambda / 2$, carries the down converter.

\subsubsection{Measurement Environment and Procedure}

The indoor measurement was performed on the $2 n d$ floor in the Micron Engineering Building at Boise State University. Fig. 3.12a illustrates the measurement environment. The measurement took place between the cubicle and offices. The wall on the office is drywall and the cubicle panels are made from fiber and foam. At the time of measurement, there were few people in the area, as shown in fig. 3.11. Outdoor measurement was performed at the Boise airport, in the same area as the Large scale measurement in section 3.1. The TX was placed in a fixed location as the RX moved to two LOS locations and one NLOS location, as shown in 3.12b. Note that during the measurement, there are aircraft arriving and departing next to the measurement location connected with the jet bridge, also, there was a truck blocking the NLOS TX-RX link at the time of measurement.

For both indoor and outdoor measurements, both TX RX antennas were placed $1.47 \mathrm{~m}$ above the floor with $0^{\circ}$ elevation angle. TX-RX separation of indoor LOS measurement is $3.9 \mathrm{~m}$, separation of outdoor LOS measurements are $7.4 \mathrm{~m}$ and $10 \mathrm{~m}$, and $8.5 \mathrm{~m}$ for outdoor NLOS measurement. The RX down converter moves 5 positions on the linear rail that is separated by half wavelength. At each linear rail position, RX PTU will point to azimuth angles from $-160^{\circ}$ to $160^{\circ}$ with a stepsize of $10^{\circ}$, as TX antenna remains at $0^{\circ}$, which is pointing at RX antenna. At each of these linear rail locations and angle combinations, 5 PDP is captured. Note that the angle is TX 


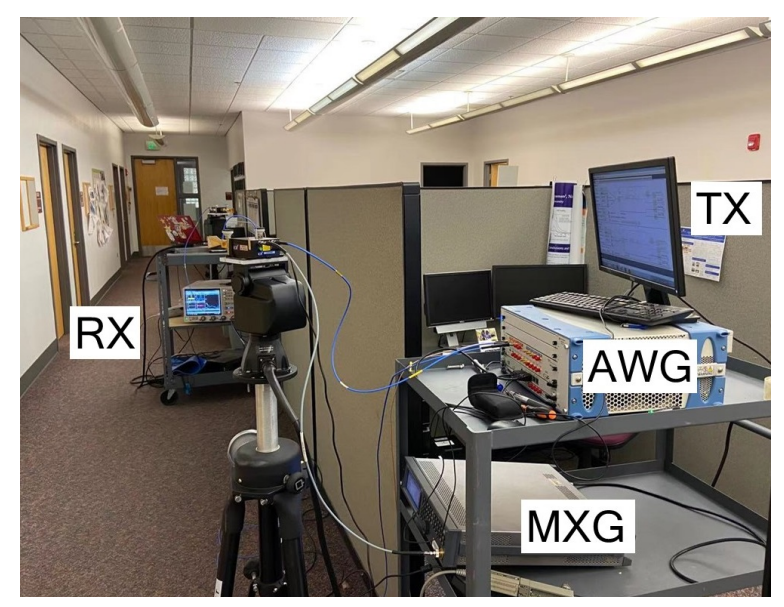

Figure 3.11: image of $73 \mathrm{GHz}$ small scale indoor measurement

and RX relevant to the LOS point to point position. The TX and RX are aligned to be LOS when both of them are at $0^{\circ}$. The positive angle is clockwise and the negative angle is counter-clockwise, as shown in Fig. 3.12. Thus, for each TX-RX separation, 33 RX pointing angles each with 5 PDPs, a total of 165 PDPs are captured. Fig. 3.13a shows different PDP obtained at different RX pointing angles at $7.4 \mathrm{~m}$ separations with $73 \mathrm{GHz}$ signal at LOS condition. It shows as the RX antenna is pointing away from the TX antenna, the magnitude of PDP is affected significantly, the dominant multipath component decreases by 20dB. Fig. 3.13b shows the angular profile at 7.4 m separation with $73 \mathrm{GHz}$ signal at LOS condition. It shows the received power varies with different RX antenna pointing angles, the received power is calculated with 3.1.

\subsubsection{Data Analysis Result}

Small scale fading describes the random fluctuations of amplitudes of multipath components. In this study, the random fluctuations come from movements of several half wavelengths and different pointing angles of antenna. The small-scale spatial fading distributions were computed with all individual multipath components over 


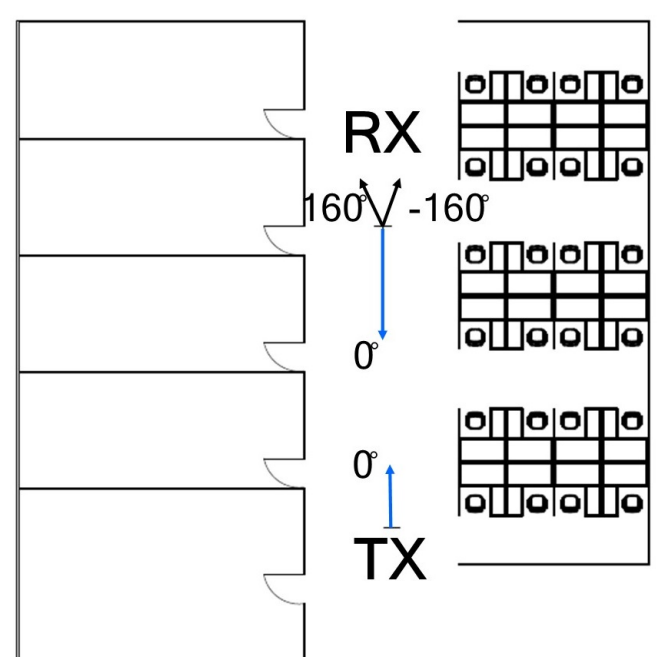

(a)

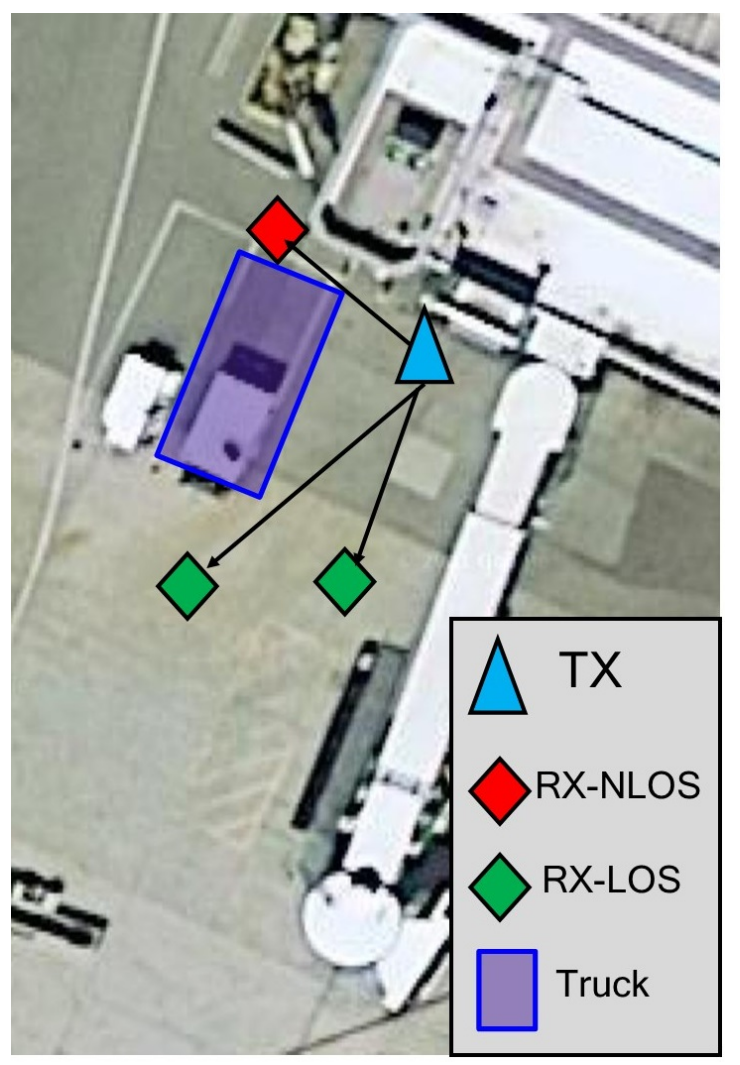

(b)

Figure 3.12: a: Layout of the small scale measurement office environment, showing office and cubicles. b: Layout of the small scale measurement outdoor environment, not showing the airplane connected to the jetbridge during the measurement, and a truck blocking the NLOS TX-RX link.

the local area with all different antenna pointing angles. The multipath components were obtained by taking local maximum within 7 samples, or within 3.6 ns bin. The noise floor is defined as $15 \mathrm{~dB}$ lower than LOS amplitude, all multipath components higher than the noise floor are included. At a TX-RX angle combination, all multipath components at 5 linear track position are grouped into one data set.

Amplitude distributions about the mean were extracted and estimated with Rayleigh, Ricean, and log-normal distribution. The Rayleigh distribution characterizes a case where the multipath components have small variations in amplitudes. Its PDF is 


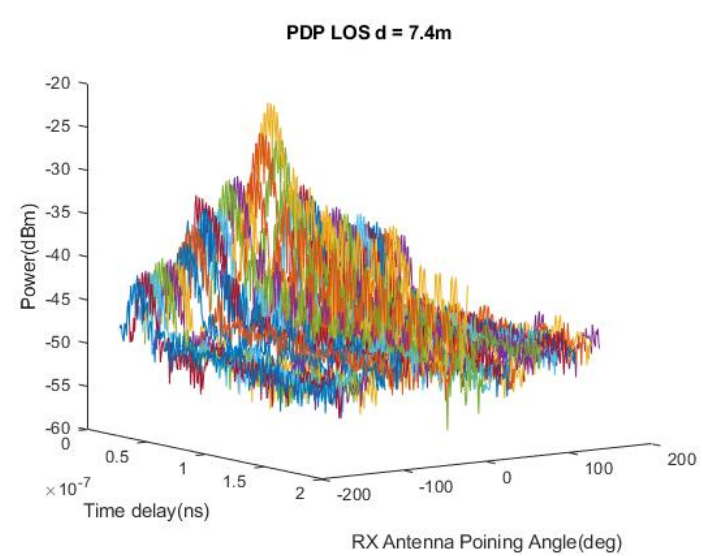

(a)

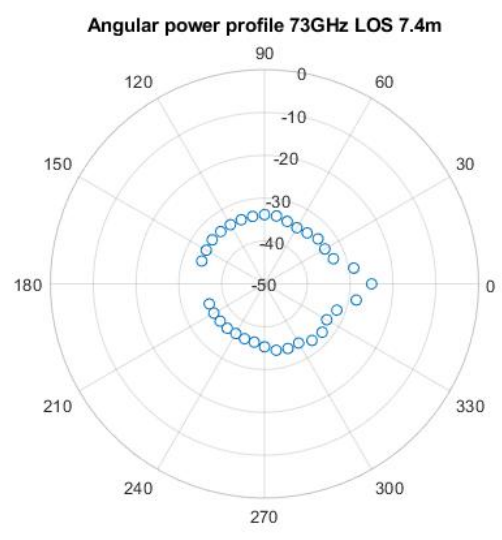

(b)

Figure 3.13: a: Power delay profiles with various RX antenna pointing angle, outdoor LOS at Boise Airport, $\mathrm{fc}=73 \mathrm{GHz} d=7.4 \mathrm{~m}$ b: Angular power profile of $73 \mathrm{GHz}$ signal, outdoor LOS at Boise Airport, $\mathrm{d}=7.4 \mathrm{~m}$

given by,

$$
p(x)=\frac{x}{\sigma^{2}} e^{-\frac{x^{2}}{2 \sigma^{2}}}
$$

where $\sigma$ is the standard deviation of multipath amplitudes. The Ricean distribution Where the PDF of Ricean distribution is given by,

$$
\begin{gathered}
p(x)=\frac{x}{\sigma^{2}} e^{-\frac{x^{2}+A^{2}}{2 \sigma^{2}}} I_{0}\left(\frac{A x}{\sigma^{2}}\right) \\
K=\frac{A^{2}}{2 \sigma^{2}}
\end{gathered}
$$

where $A$ denotes the peak amplitude of dominant signal, $I_{0}()$ is the modified Bessel function of the first kind and zero order, and $\sigma$ is the standard deviation of all other weak path amplitudes. The $\mathrm{K}$ factor is defined as the ratio between the deterministic signal power and the variance of the multipath [16]. The PDF of a log-normal 
distribution is given by,

$$
p(x)=\frac{1}{\sqrt{2 \pi} \sigma x} e^{-(\log x-\bar{x}) / 2 \sigma^{2}}
$$

where $\bar{x}$ is the mean and $\sigma$ is the standard deviation of the multipath components.

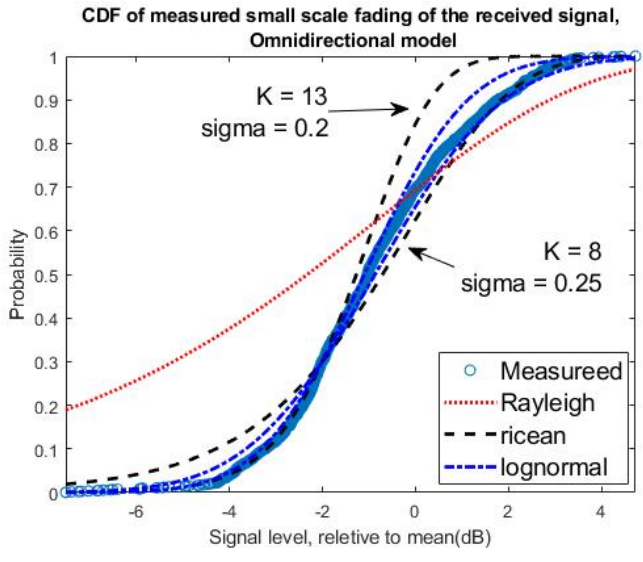

(a)

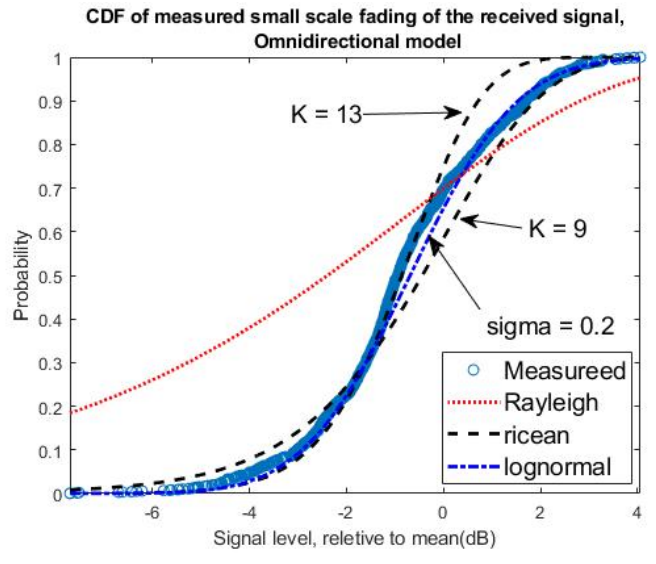

(b)

Figure 3.14: CDFs of the, a: $73 \mathrm{Ghz}$ and b: $81 \mathrm{GHz}$, small scale fading of individual path amplitude relative to rms amplitude, in LOS indoor environment.

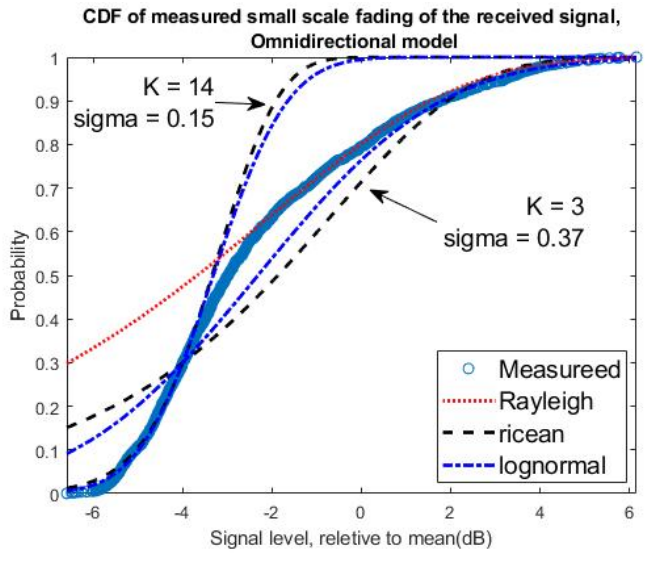

(a)

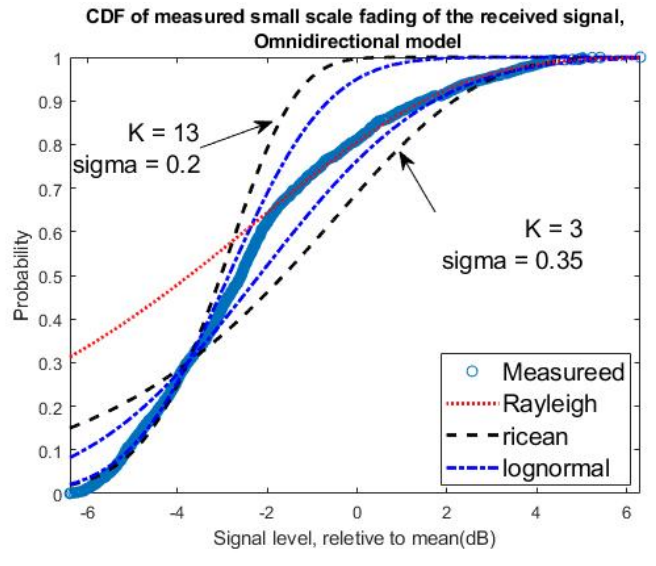

(b)

Figure 3.15: CDFs of the, a: $73 \mathrm{Ghz}$ and b: $81 \mathrm{GHz}$, small scale fading of individual path amplitude relative to rms amplitude, in LOS outdoor airport environment.

The empirical cumulative distribution functions (CDF) of $a_{k} / a_{r m s}$ for different environments and frequencies are shown in Fig. 3.14, 3.15, and 3.16, where $a_{k}$ are 


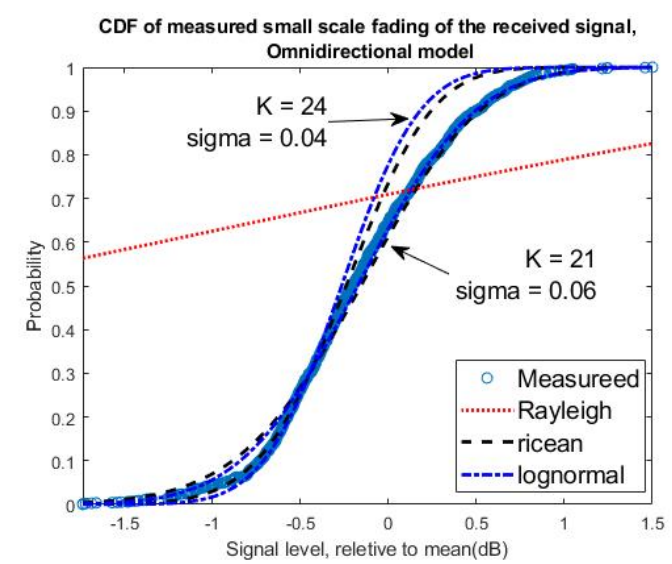

(a)

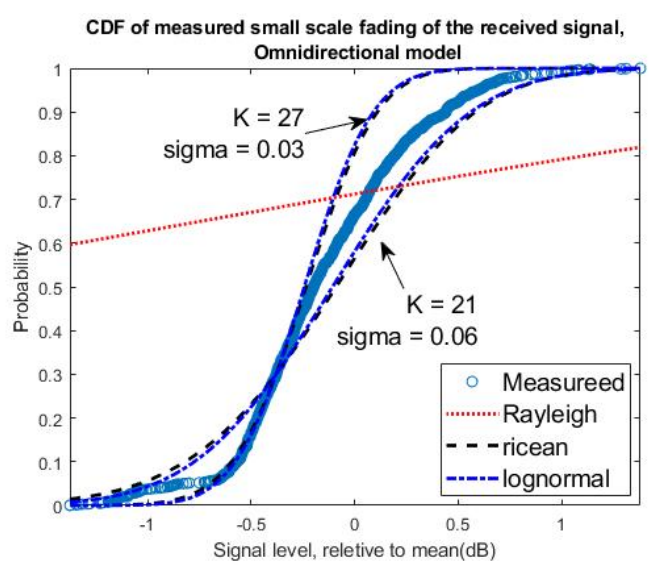

(b)

Figure 3.16: CDFs of the, a: $73 \mathrm{Ghz}$ and b: $81 \mathrm{GHz}$, small scale fading of individual path amplitude relative to rms amplitude, in NLOS outdoor airport environment.

amplitude of $k t h$ multipath components, and $a_{r m s}$ is the rms value of all multipath components. They also show the CDF of Rayleigh, Ricean and log-normal distributions.

For the LOS indoor scenario at BSU (Fig. 3.14), the $73 \mathrm{GHz}$ curve is bounded by Ricean distributions with K-factors of $8 \mathrm{~dB}$ and $13 \mathrm{~dB}$, also bounded by log-normal distribution with $\sigma$ of $0.2 \mathrm{~dB}$ and $0.25 \mathrm{~dB} .81 \mathrm{Ghz}$ shows a comparable result which is bounded by Ricean distributions with K-factors of $9 \mathrm{~dB}$ and $13 \mathrm{~dB}$, and fits closely with log-normal distribution $\sigma$ of $0.2 \mathrm{~dB}$. The power fluctuation for both frequency bands are from $-7 \mathrm{~dB}$ to $4.5 \mathrm{~dB}$. For LOS outdoor scenarios at the Boise airport (Fig. 3.14), the $73 \mathrm{GHz}$ curve is bounded by by Ricean distributions with K-factors of $3 \mathrm{~dB}$ and $14 \mathrm{~dB}$, and also bounded by log-normal distribution with $\sigma$ of $0.15 \mathrm{~dB}$ and $0.37 \mathrm{~dB}$. The $81 \mathrm{GHz}$ curve is bounded by K-factor of $3 \mathrm{~dB}$ and $13 \mathrm{~dB}$, also by $\sigma$ of $0.2 \mathrm{~dB}$ and $0.35 \mathrm{~dB}$. The power fluctuation for both frequency band are from -6.5 $\mathrm{dB}$ to $6 \mathrm{~dB}$.

For both indoor and outdoor LOS cases, the upper K-factors are around 13 to 14 
$\mathrm{dB}$, which is similar to simulation model used by 3GPP [23], which is $K=13-0.03 d$ $\mathrm{dB}$, where $d$ is the TX-RX separation in meters. The difference comes from the fluctuation of amplitude and probability of the multipath amplitude distributions. For both bands, indoor LOS shows a smaller fluctuation compared with outdoor LOS. Indoor LOS multipath amplitude show higher probability at $-3 \mathrm{~dB}$ to $1 \mathrm{~dB}$ relative to mean amplitude, as outdoor LOS multipath amplitude at $-5 \mathrm{~dB}$ to -2 $\mathrm{dB}$. This difference is due to the indoor environment is rich scatter and rich multipath from walls and cubicles, compared with the outdoor airport tarmac which has fewer obstacles.

For the NLOS outdoor scenario at the Boise airport, the $73 \mathrm{GHz}$ curve is bounded by Ricean distributions with K-factors of $21 \mathrm{~dB}$ and $24 \mathrm{~dB}$, also bounded by lognormal distribution with $\sigma$ of $0.04 \mathrm{~dB}$ and $0.06 \mathrm{~dB}$. $81 \mathrm{GHz}$ shows a comparable result which is bounded by Ricean distributions with K-factors of $21 \mathrm{~dB}$ and $27 \mathrm{~dB}$, and bounded log-normal distribution $\sigma$ of $0.03 \mathrm{~dB}$ and $0.06 \mathrm{~dB}$. The power fluctuation for both frequency bands is around $-1.5 \mathrm{~dB}$ to $1.5 \mathrm{~dB}$, the $73 \mathrm{GHz}$ band shows a slightly higher fluctuation. Note that during NLOS measurement, only one RX angle out of 33 has a weak received signal as the rest angle shows no signal, which results in a low standard deviation of multipath amplitudes. A K-factor of 21 also predicts more occurrences of deep fading for this scenario.

Overall, the CDF varies insignificantly between indoor LOS to outdoor LOS scenarios and varies significantly from LOS to NLOS scenarios. Also, CDF of $73 \mathrm{GHz}$ and $81 \mathrm{GHz}$ signal are comparable. For all measurement environments and scenarios, log-normal distribution is showing better fits than Ricean distribution, lastly, Rayleigh distribution fits poorly with all CDFs. 


\section{CHAPTER 4}

\section{CONCLUSION}

In this thesis, I presented measurement campaigns that were conducted at the Boise State University and Boise Airport at $73 \mathrm{GHz}$ and $81 \mathrm{GHz}$ to investigate the properties of mmWave channels. The large scale channel parameters were estimated with the close-in reference model and floating-intercept model. Our work shows that the LOS CI PLEs are close to the theoretical $\operatorname{PLE}(n=2)$ of the free space model, and NLOS CI PLEs are close to $\operatorname{PLE}(n=4)$ of the free space model. The FI model provides a better fit for measured data. The standard deviation $(\sigma)$ is higher at the airport indoors environment than outdoors on the tarmac, due to more obstacles appearing indoors, which creates a more reflective environment. Building material attenuation measurement at $73 \mathrm{GHz}$ band is also presented, along with work at $28 \mathrm{GHz}$ from NCSU, and work at $91 \mathrm{GHz}$ from USC. Clear glass, cinder block, plywood, drywall, and acoustic ceiling tiles were tested. The measurement was designed to minimize signal distortion, e.g. reflections, and multi-path components, caused by other objects in the environment. Nine measurement locations were chosen for both wideband and narrowband measurements to reduce the effects from misalignment and material inhomogeneity. With the same material, the highest attenuation is always at $91 \mathrm{GHz}$. Clear glass and plywood provide the most difference on attenuation at three different bands. In addition, small-scale spatial fading measurement at Boise State University 
and the Boise airport is presented. The measurement shows the effects on the received signal from different antenna pointing angles, from $-160^{\circ}$ to $160^{\circ} \mathrm{RX}$. Moreover, we model the omnidirectional small scale fading with Rayleigh, Ricean, and Log-normal distribution models. Indoor LOS scenario Ricean K-factor is from $13 \mathrm{~dB}$ to $8 \mathrm{~dB}$, and Log-normal $\sigma$ is from $0.2 \mathrm{~dB}$ to $0.25 \mathrm{~dB}$. Outdoor LOS scenario Ricean K-factor is from $13 \mathrm{~dB}$ to $3 \mathrm{~dB}$, and Log-normal $\sigma$ is from $0.15 \mathrm{~dB}$ to $0.37 \mathrm{~dB}$. Outdoor NLOS scenario Ricean K-factor is from $21 \mathrm{~dB}$ to $27 \mathrm{~dB}$, and Log-normal $\sigma$ is from $0.03 \mathrm{~dB}$ to $0.06 \mathrm{~dB}$.Our result can help designers to estimate path loss, fading, and building material attenuation of the mmWave system.

Future work includes modeling more small scale parameters, e.g angular spread, rms delay. 


\section{REFERENCES}

[1] M. Khatun, "Millemeter-wave channel measurement for airport environments," Ph.D. dissertation, 2019.

[2] N. Hosseini, M. Khatun, C. Guo, K. Du, O. Ozdemir, D. Matolak, I. Guvenc, and H. Mehrpouyan, "Attenuation of several common building materials in millimeter-wave frequency bands: 28, 73 and 91ghz," IEEE Antenna and Propagation Magazine, 2020.

[3] T. S. Rappaport, R. W. Heath Jr, R. C. Daniels, and J. N. Murdock, Millimeter wave wireless communications. Englewood Cliffs, NJ, USA: Prentice Hall, 2015.

[4] F. Rusek, D. Persson, B. K. Lau, E. G. Larsson, T. L. Marzetta, O. Edfors, and F. Tufvesson, "Scaling up mimo: Opportunities and challenges with very large arrays," IEEE Signal Processing Magazine, vol. 30, no. 1, pp. 40-60, 2013.

[5] M. Wen, B. Zheng, K. J. Kim, M. Di Renzo, T. A. Tsiftsis, K. Chen, and N. Al-Dhahir, "A survey on spatial modulation in emerging wireless systems: Research progresses and applications," IEEE Journal on Selected Areas in Communications, vol. 37, no. 9, pp. 1949-1972, 2019.

[6] T. S. Rappaport, S. Sun, R. Mayzus, H. Zhao, Y. Azar, K. Wang, G. N. Wong, J. K. Schulz, M. Samimi, and F. Gutierrez, "Millimeter wave mobile communications for $5 \mathrm{~g}$ cellular: It will work!" IEEE Access, vol. 1, pp. 335-349, 2013.

[7] H. Zhao, R. Mayzus, S. Sun, M. Samimi, J. K. Schulz, Y. Azar, K. Wang, G. N. Wong, F. Gutierrez, and T. S. Rappaport, "28 ghz millimeter wave cellular communication measurements for reflection and penetration loss in and around buildings in new york city," in 2013 IEEE International Conference on Communications (ICC), 2013, pp. 5163-5167.

[8] G. R. MacCartney, M. K. Samimi, and T. S. Rappaport, "Omnidirectional path loss models in new york city at 28 ghz and 73 ghz," in 2014 IEEE 25th Annual International Symposium on Personal, Indoor, and Mobile Radio Communication (PIMRC), 2014, pp. 227-231. 
[9] T. S. Rappaport, G. R. MacCartney, M. K. Samimi, and S. Sun, "Wideband millimeter-wave propagation measurements and channel models for future wireless communication system design," IEEE Transactions on Communications, vol. 63, no. 9, pp. 3029-3056, 2015.

[10] S. Sun, G. R. MacCartney, and T. S. Rappaport, "A novel millimeter-wave channel simulator and applications for 5g wireless communications," in 2017 IEEE International Conference on Communications (ICC), 2017, pp. 1-7.

[11] S. Sun, H. Yan, G. R. MacCartney, and T. S. Rappaport, "Millimeter wave small-scale spatial statistics in an urban microcell scenario," in 2017 IEEE International Conference on Communications (ICC), 2017, pp. 1-7.

[12] D. W. Matolak, M. Mohsen, and J. Chen, "Path loss at 5 ghz and 31 ghz for two distinct indoor airport settings," in 2019 27th European Signal Processing Conference (EUSIPCO), 2019, pp. 1-5.

[13] M. R. Akdeniz, Y. Liu, M. K. Samimi, S. Sun, S. Rangan, T. S. Rappaport, and E. Erkip, "Millimeter wave channel modeling and cellular capacity evaluation," IEEE Journal on Selected Areas in Communications, vol. 32, no. 6, pp. 11641179, 2014.

[14] H. T. Friis, "A note on a simple transmission formula," Proceedings of the IRE, vol. 34, no. 5, pp. 254-256, 1946.

[15] M. Khatun, C. Guo, D. Matolak, and H. Mehrpouyan, "Indoor and outdoor penetration loss measurements at 73 and $81 \mathrm{ghz}$," in 2019 IEEE Global Communications Conference (GLOBECOM), 2019, pp. 1-5.

[16] T. S. Rappaport, R. W. Heath Jr, R. C. Daniels, and J. N. Murdock, Wireless communications priciples and practice. Englewood Cliffs, NJ, USA: Prentice Hall, 2015.

[17] M. Khatun, C. Guo, L. Moro, D. Matolak, and H. Mehrpouyan, "Millimeter-wave path loss at $73 \mathrm{ghz}$ in indoor and outdoor airport environments," in 2019 IEEE 90th Vehicular Technology Conference (VTC2019-Fall), 2019, pp. 1-5.

[18] D. W. Matolak, M. Mohsen, and J. Chen, "Path loss at 5 ghz and 31 ghz for two distinct indoor airport settings," in 2019 27th European Signal Processing Conference (EUSIPCO), 2019, pp. 1-5.

[19] G. R. MacCartney, Junhong Zhang, Shuai Nie, and T. S. Rappaport, "Path loss models for $5 \mathrm{~g}$ millimeter wave propagation channels in urban microcells," in 2013 IEEE Global Communications Conference (GLOBECOM), 2013, pp. 3948-3953. 
[20] K. Pahlavan and A. H. Levesque, Wireless Information Networks (Wiley Series in Telecommunications and Signal Processing). Hoboken, NJ, USA: John Wiley Sons, Inc., 2005.

[21] S. Sun, G. R. MacCartney, and T. S. Rappaport, "Millimeter-wave distancedependent large-scale propagation measurements and path loss models for outdoor and indoor 5g systems," in 2016 10th European Conference on Antennas and Propagation (EuCAP), 2016, pp. 1-5.

[22] W. Stone, "Electromagnetic Signal Attenuation in Construction Materials," National Institute of Standards and Technology, Report 6055, 1997.

[23] ETSI, "Universal mobile telecommunications system (umts); spatial channel model for multiple input multiple output (mimo) simulations," 2007. 


\section{Publications}

1. Nozhan Hosseini, Mahfuza Khatun, Changyu Guo, Kairui Du, Ozgur Ozdemir, David W. Matolak, Ismail Guvenc, Hani Mehrpouyan, "Attenuation of Several Common Building Materials in Millimeter-Wave Frequency Bands: 28, 73 and 91 GHz" IEEE Antennas and Propagation Magazine, 2020.(Accepted)

2. Mahfuza Khatun, Changyu Guo, Hani Mehrpouyan, "Penetration and Reflection Characteristics in Millimeter-Wave Indoor Channels" 2020 International Conference on Electromagnetic in Advanced Applications(ICEAA '20), 2020.(Accepted)

3. Mahfuza Khatun, Changyu Guo, David Matolak, Hani Mehrpouyan, "Indoor and Outdoor Penetration Loss Measurements at 73 and 81 GHz" 2019 IEEE Global Communications Conference (GLOBECOM), 2019.

4. Mahfuza Khatun, Changyu Guo, Letizia Moro, David Matolak, Hani Mehrpouyan, "Millimeter-Wave Path Loss at $73 \mathrm{GHz}$ in Indoor and Outdoor Airport Environments" 2019 IEEE 90th Vehicular Technology Conference: VTC2019-Fall, 2019. 
APPENDIX A

LINEAR RAIL SYSTEM 
This system contains four parts. Fuyu FL80 Linear rail, ST-M5045 stepper motor controller, AC-DC buck converter, and Arduino UNO microcontroller. Fig.A.1b shows the block diagram of the Linear rail system. The AC-DC buck converter output $24 \mathrm{~V} \mathrm{DC}$ voltage to power the Linear rail motor and the motor controller. The Arduino is programmed to send out pulses, with a push of a button on a breadboard, to the motor controller which drives the motor in linear rail.

The Arduino is programmed to send out 640 pulses with a single push of a button, speed of motion, and the movement distance per button push, can be varied by modifying the pulse. With the current setting on the motor driver, each pulse moves the linear rail $0.032 \mathrm{~mm}$. Motor driver settings can be changed for more resolution options.

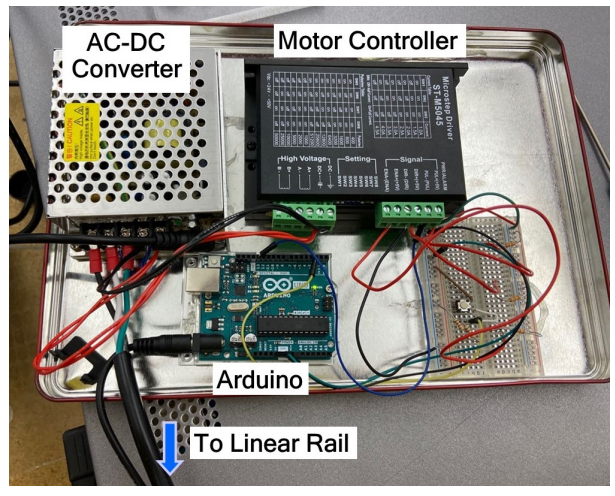

(a) Linear Rail Control module setup

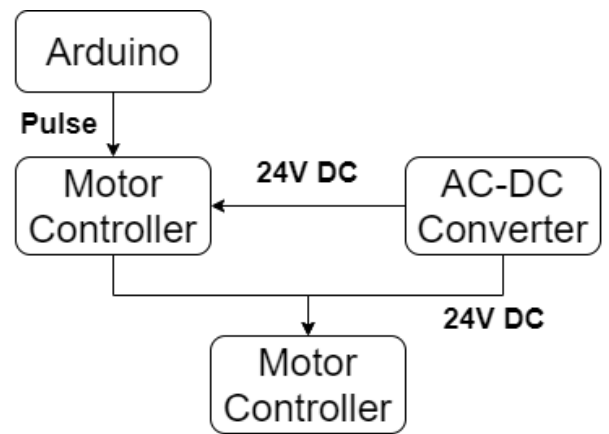

(b) Linear Rail System Block Diagram

Figure A.1: Linear Rail Module 


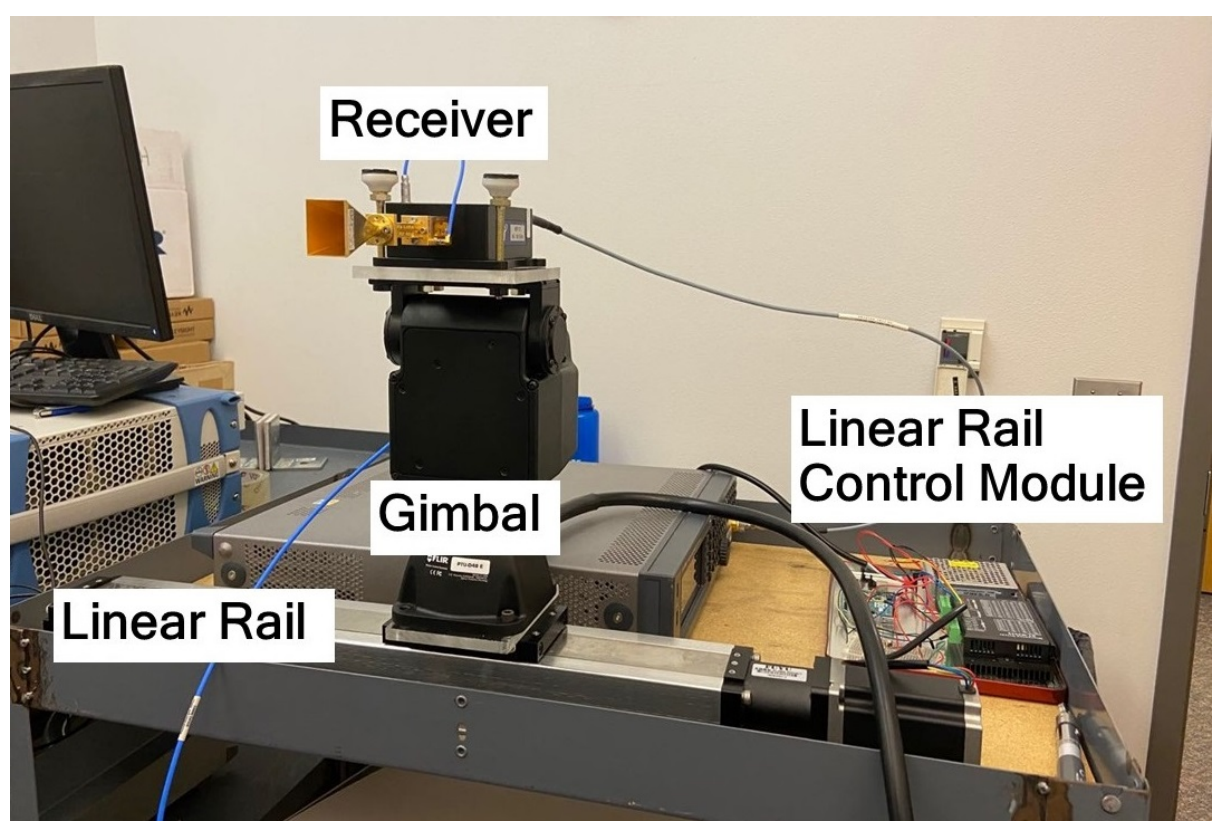

Figure A.2: Linear rail with Gimbal and RX mounted on cart 
APPENDIX B

PAN-TILT UNIT 
The Pan-Tilt Unit is controlled by laptops. Each PTU is connected to a laptop with SR-232 to usb cable. The control code is written in MatLab, integrated with data recording SCPI code. Two laptops are connected through UTP on WLAN. Fig. B.1 shows the block diagram of the PTU control system.

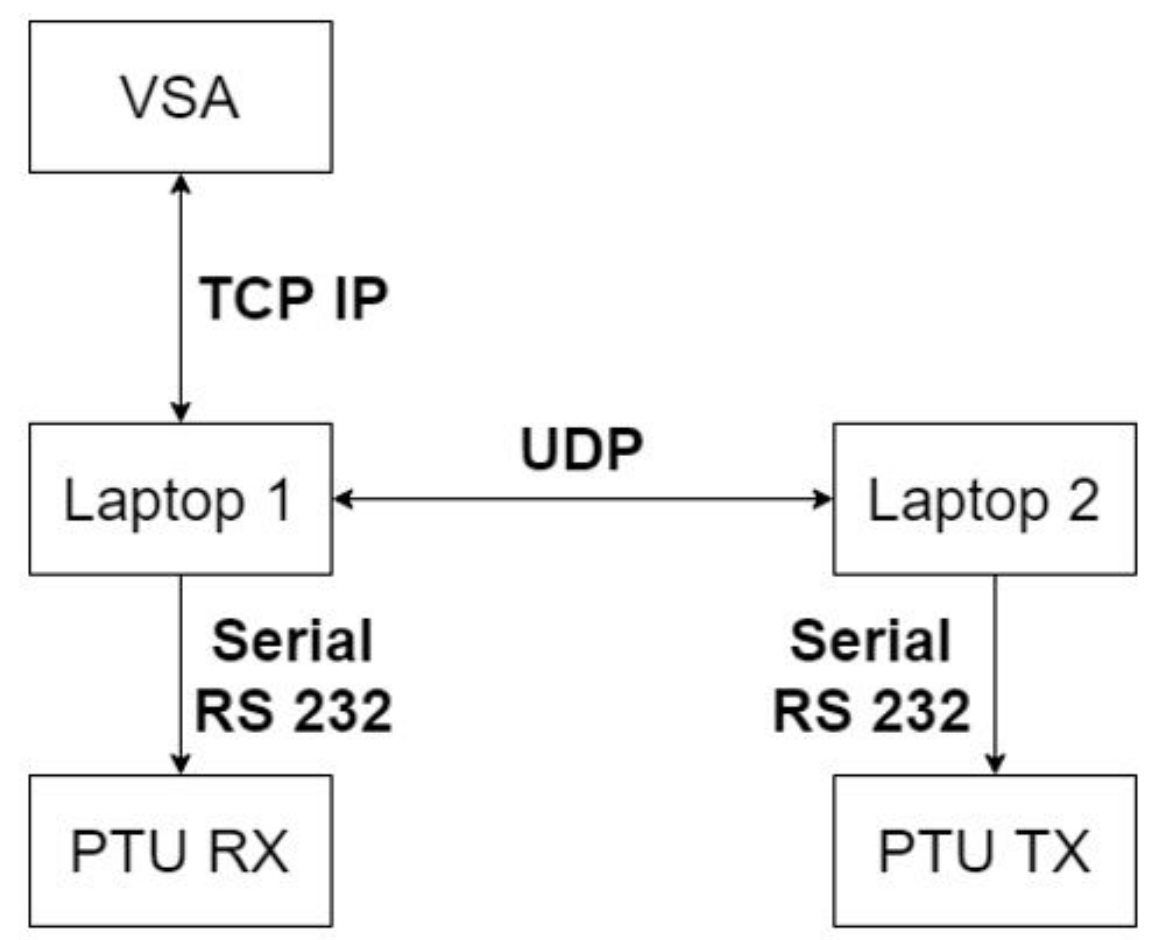

Figure B.1: PTU Control System Diagram

The control code in MatLab contains PTU control and VSA data recording functionality. Fig. B.2 shows the control flow of the MatLab script. The RX control script starts first and then the TX control script. The TX script will control the PTU move to a designated angle then send a done message to the UDP buffer. When the RX control script receives the done message, it moves the PTU then captures data from VSA software. After the data capture finishes, a done message is sent to the UPD buffer as TX is waiting. 

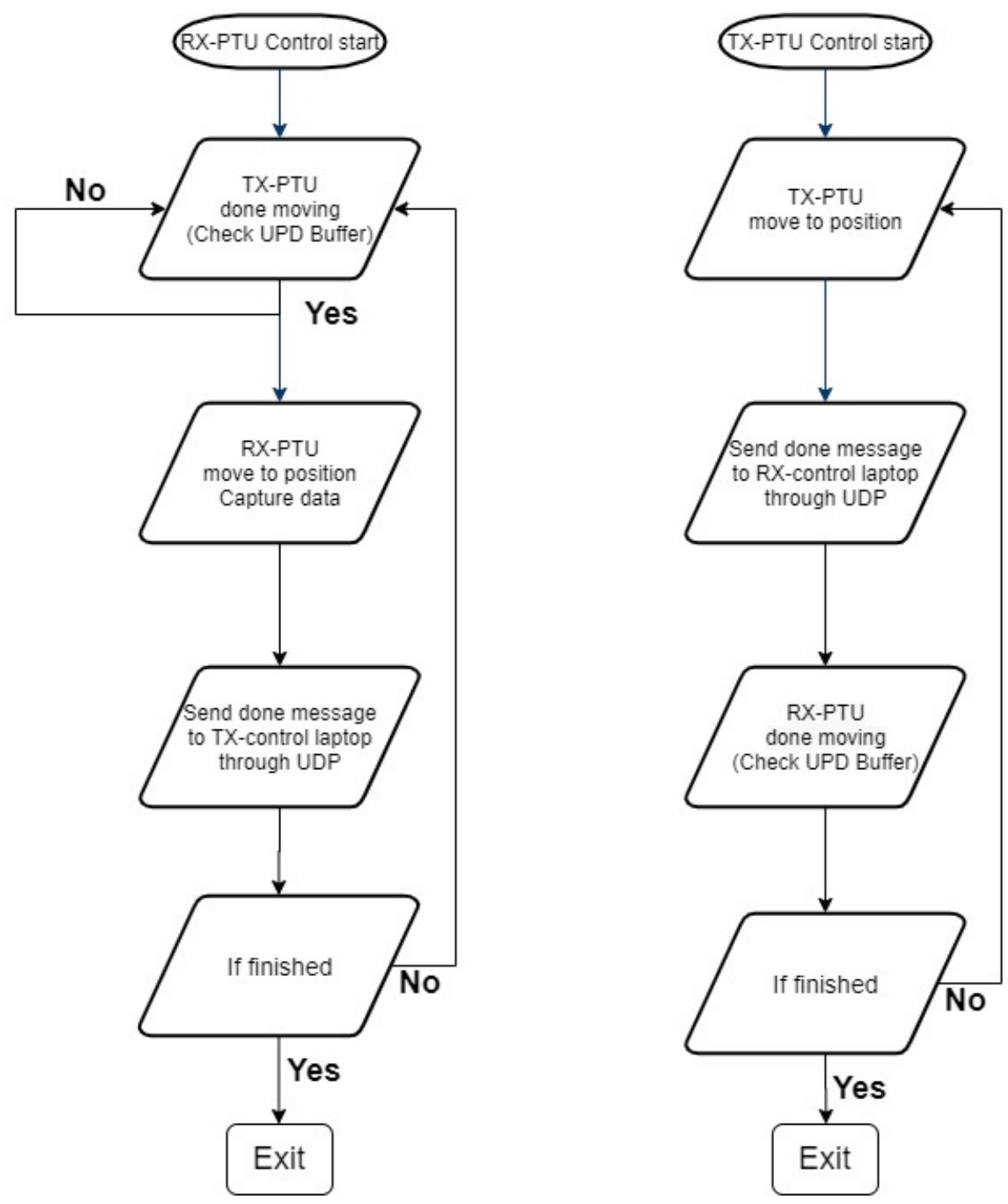

Figure B.2: PTU Control flow 


\section{APPENDIX C}

\section{ADDITIONAL FIGURES FOR MATERIALS ATTENUATION MEASUREMENT}



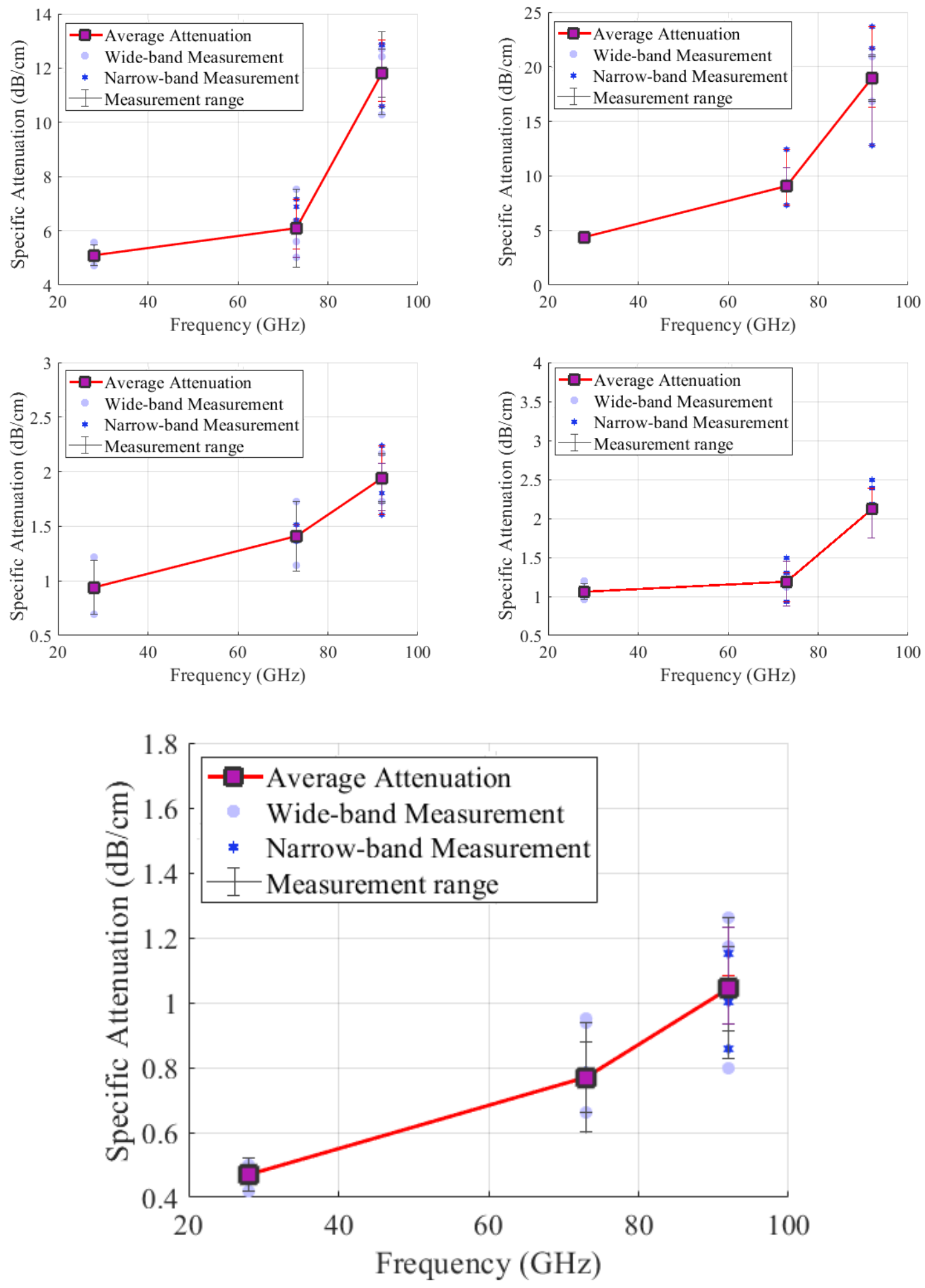

Figure C.1: Specific Attenuation versus Frequency for different materials. top left: plywood, top right: glass, middle left: drywall, middle right: concrete, bottom: ceiling tile $[2]$ 